\title{
Rapidly Exponentially Stabilizing Control Lyapunov Functions and Hybrid Zero Dynamics
}

\author{
Aaron D. Ames, Kevin Galloway, J. W. Grizzle, and Koushil Sreenath
}

\begin{abstract}
This paper addresses the problem of exponentially stabilizing periodic orbits in a special class of hybrid modelssystems with impulse effects-through control Lyapunov functions. The periodic orbit is assumed to lie in a $C^{1}$ submanifold $Z$ that is contained in the zero set of an output function and is invariant under both the continuous and discrete dynamics; the associated restriction dynamics are termed the hybrid zero dynamics. The orbit is furthermore assumed to be exponentially stable within the hybrid zero dynamics. Prior results on the stabilization of such periodic orbits with respect to the fullorder dynamics of the system with impulse effects have relied on input-output linearization of the dynamics transverse to the zero dynamics manifold. The principal result of this paper demonstrates that a variant of control Lyapunov functions that enforce rapid exponential convergence to the zero dynamics surface, $Z$, can be used to achieve exponential stability of the periodic orbit in the full-order dynamics, thereby significantly extending the class of stabilizing controllers. The main result is illustrated on a hybrid model of a bipedal walking robot through simulations and is utilized to experimentally achieve bipedal locomotion via control Lyapunov functions.
\end{abstract}

\section{INTRODUCTION AND Motivation}

For finite-dimensional nonlinear systems, exhibiting a Lyapunov function has been the gold standard for establishing stability properties of equilibrium points. Indeed, under mild conditions, the existence of a Lyapunov function with particular properties is equivalent to stability in the sense of Lyapunov, asymptotic stability, or exponential stability of an equilibrium point [23], [39]. Moreover, once a Lyapunov function has been found, a region of attraction can be estimated; albeit, the estimate is often very conservative. Lyapunov functions have also been used to define and characterize input-to-state stability [40], [41]. Recently, Lyapunov functions have seen a further surge of interest in the nonlinear systems community through powerful tools for obtaining them as sum of squares [28], [45]. All of this points to the power of Lypunov functions in understanding nonlinear systems.

The work of A. D. Ames is supported by NSF grants CNS-0953823 and CNS-1136104, NHARP project 000512-0184-2009 and NASA contract NNX12AB58G, K. Galloway is supported by DARPA Contract W91CRB11-1-0002, and J. Grizzle is supported in part by DARPA and by NSF grant ECS-909300.

A. D. Ames is with the Department of Mechanical Engineering, Texas A\&M University, College Station, TX 77843, email: aames @ t amu . edu.

K. Galloway and J. W. Grizzle are with the Control Systems Laboratory, Electrical Engineering and Computer Science Department, University of Michigan, Ann Arbor, MI 48109, email: \{kevinsg,grizzle\}@umich.edu.

K. Sreenath is with the GRASP Laboratory, Department of Mechanical Engineering and Applied Mechanics, University of Pennsylvania, Philadelphia, PA 19104, email: koushils@seas.upenn.edu.
In the context of nonlinear control systems, since the ground-breaking work of Artstein [5] and Sontag [38] control Lyapunov functions have become central to feedback design. Roughly speaking, given a nonlinear control system with equilibrium point at the origin,

$$
\dot{x}=f(x, u),
$$

a positive definite function $V(x)$ is a control Lyapunov function if, for each $x \neq 0$ in the state space, there exists $u$ such that

$$
\dot{V}(x, u)=\operatorname{grad} V(x) f(x, u)<0 .
$$

Domains of application include nonlinear adaptive control [24], robust nonlinear feedback design [33], receding horizon control of nonlinear systems [22], and stabilization of hybrid systems [31] to name only a few. An important aspect of using the control Lyapaunov approach is that the designer is free to choose among an infinite number of feedback control laws that render the time derivative of the Lyapunov function negative definite. In particular, reference [10] proposed for control affine nonlinear systems, the so-called "point-wise minimum norm" controller, which at each instant of time selects the control value of minimum norm among all values rendering " $\dot{V}$ " negative definite; moreover, if the set where the control takes values is convex, then this controller is Lipschitz continuous. The point-wise minimum norm controller is optimal with respect to a cost function [10]; therefore, control Lyapunov functions not only establish proofs of stability for nonlinear control systems through the generation of a family of controllers, but simultaneously have a direct relationship with optimality.

The primary goal of this paper is to extend the control Lyapunov methodology to a class of hybrid systems that includes bipedal robots. The work is motivated by the practical problem of enlarging the class of feedback controllers that can create exponentially stable periodic walking gaits; such gaits correspond to exponentially stable periodic orbits in a class of systems with impulse effects. In the context of the overarching goal, and motivated by the application of robotic locomotion, this work presents three main novel results: extending control Lyapunov functions to allow direct control over the rate of exponential convergence to a (hybrid) zero dynamics surface; proving that exponentially stabilizing control Lyapunov functions allow for the extension of the stable periodic orbits in the (hybrid) zero dynamics to stable periodic orbits in the fullorder dynamics; and finally demonstrating these concepts both in simulation and experimentally on bipedal walking robots. 
In order to put these results in context, it is necessary to review hybrid zero dynamics and the current paradigm for constructing controllers relative to the zero dynamics manifold. The notion of hybrid zero dynamics (HZD) was introduced in [47] as a feedback design method that could accommodate underactuation in bipedal robots and thereby move beyond quasi-static, flat-footed walking gaits. At its most basic level, the hybrid zero dynamics is an extension of the Byrnes-Isidori notion of zero dynamics [21], [20] to a hybrid setting, with the additional requirement that the invariant manifolds on which the zero dynamics is defined must also be invariant under the discrete reset maps. Outputs satisfying a vector relative degree [20] condition are designed for each phase of a hybrid model with the objective of creating an exponentially stable periodic orbit in the hybrid zero dynamics [47], [48], [49], [43]. The feedback design is completed by rendering the zero dynamics manifold sufficiently rapidly exponentially attractive [26], [49], [25]. The motivation for assuring a sufficiently fast rate of exponential convergence is that the reset maps can be (and in practice, often are) "expansive", taking a solution that does not lie in the zero dynamics manifold and "pushing" it "further away" from the manifold. Hence, the convergence of the continuous dynamics to the manifold must be sufficiently "attractive" to overcome the "repulsion" of the reset map.

To date, the design of the controller assuring convergence to the zero dynamics manifold has been approached in three ways. In [13], Hölder continuous feedbacks based on [8] were used to assure finite-time convergence. In [34], [35], specially selected variables transverse to the zero dynamics manifold are Jacobian linearized and an exponentially stabilizing feedback is computed on the basis of LQR for periodic systems (see also [7] and [36]). In [1], [2], [3], [25], [26], [49] (to only name a few), the transverse dynamics were input-output linearized and a high-gain time-invariant linear feedback was then employed; for example, when the outputs have vector relative degree 2 , after creating a linear relationship between the inputs and outputs, the feedback is chosen to be

$$
u=-\frac{1}{\varepsilon^{2}} K_{P} y-\frac{1}{\varepsilon} K_{D} \dot{y} .
$$

Therefore, the vast body of work that utilizes hybrid zero dynamics for controller design essentially considers PD control on the outputs.

The objective of the present paper is to significantly expand the set of feedback controllers that can be used to render the zero dynamics sufficiently rapidly attractive to exponentially stabilize the periodic orbit in the full-dimensional hybrid model. A control Lyapunov function (CLF) approach is taken, starting with a review of CLFs for periodic orbits of nonhybrid systems in Section II. Section III then revisits inputoutput linearization from a Lyapunov perspective and subsequently presents a CLF approach to obtaining exponential convergence bounds similar to the linear case. This section culminates with the derivation of the control (38), which is based on Sontag's construction. In Section IV we address the more general setting of affine control systems with an invariant zero dynamics surface. The goal is to analyze the full system dynamics in terms of the reduced system dynamics coupled with a CLF which converges sufficiently rapidly in the transverse dynamics. Such a CLF can be used in conjunction with the pointwise min-norm control described in (45). The main result of this paper, presented in Section $\mathrm{V}$ and proved in Section VI, applies the CLF approach in the context of hybrid systems. In particular, we show that for a CLF that is rapidly exponentially stabilizing, the existence of an exponentially stable periodic orbit in the hybrid zero dynamics implies the existence of a feedback rendering the periodic orbit exponentially stable in the full dynamics. The key difference with [31] is that asymptotic stability of the zero dynamics is not strong enough to achieve stability of the closed-loop system with the type of reset maps encountered in bipedal locomotion. Finally, Section VII describes simulation results for the CLF-based controllers implemented on a three-link bipedal walker model and Section VIII reports on experimental results.

The results presented in this paper were initially reported in [4]. The present paper adds to the original contribution in the following important ways: the non-hybrid case is fully developed through Theorem 1; the proof of the main resultTheorem 2-is carried out in full while it was omitted from [4]; and the theoretical results are verified experimentally. These factors combine to create a complete exposition on the application of control Lyapunov functions to hybrid zero dynamics with application to bipedal robotic locomotion.

\section{Exponentially Stabilizing CLFs And PERIOdic ORBITS}

The goal of this section is to provide conditions for establishing the exponential stability of a periodic orbit of a system on the basis of two lower-dimensional problems, specifically, establishing the exponential stability of a periodic orbit contained in an invariant submanifold and constructing a control Lyapunov function for the dynamics transverse to the submanifold. The problem of stabilizing a periodic orbit was first posed in these terms in [16]. We begin by considering an affine control system of the form

$$
\begin{aligned}
\dot{x} & =f(x, z)+g(x, z) u \\
\dot{z} & =q(x, z),
\end{aligned}
$$

where, $x \in X$ are the controlled (or output) states, $z \in Z$ are the uncontrolled states, $U$ is the set of admissible control values for $u$, and $X, Z$, and $U$ are open subsets of appropriately dimensioned Euclidean spaces. The vector fields $f, g$, and $q$ are assumed to be locally Lipschitz continuous. In addition, we assume that $f(0, z)=0$, so that the surface $Z$ defined by $x=0$ with dynamics $\dot{z}=q(0, z)$ is invariant.

Because systems having the form of (1) naturally arise in the study of systems with zero dynamics [20], $Z$ will be referred to as the zero dynamics manifold and the reduced system as the zero dynamics. The $x$-dynamics are sometimes called the transverse dynamics. Models having the form of (1) also arise in the study of underactuated mechanical systems (i.e., fewer inputs than degrees of freedom) [42], [30], [15], where the configuration variables are split into "actuated" and 
"unactuated" coordinates. Moreover, the actuated configuration variables can be interpreted as a set of outputs with vector relative degree 2 [20], meaning the inputs show up in the second derivative of the outputs, and the matrix multiplying the inputs, called the decoupling matrix, is square and full rank in a neighborhood of interest. In this case, the unactuated configuration variables and their first derivatives constitute the zero dynamics and the actuated variables and their first derivatives constitute the transverse dynamics.

Definition 1: (Based on [51, Def. 3, pp. 4] for discretetime systems.) For the system (1), a continuously differentiable function $V: X \rightarrow \mathbb{R}$ is an exponentially stabilizing control Lyapunov function (ES-CLF) if there exists positive constants $c_{1}, c_{2}, c_{3}>0$ such that

$$
\begin{aligned}
& c_{1}\|x\|^{2} \leq V(x) \leq c_{2}\|x\|^{2} \\
& \inf _{u \in U}\left[L_{f} V(x, z)+L_{g} V(x, z) u+c_{3} V(x)\right] \leq 0
\end{aligned}
$$

for all $(x, z) \in X \times Z$.

We are interested in how the zero dynamics and an ESCLF allow us to understand the stability of periodic orbits. To make the discussion precise, some elementary definitions and notation are recalled.

Motivated by the constructions in [10], we define the set

$$
K(x, z)=\left\{u \in U: L_{f} V(x, z)+L_{g} V(x, z) u+c_{3} V(x) \leq 0\right\}
$$

consisting of the control values that result in $\dot{V}(x, z, u) \leq$ $-c_{3} V(x)$. When $V$ is an ES-CLF, it is easy to see that for any locally Lipschitz continuous feedback control law $u(x, z)$ such that $u(x, z) \in K(x, z)$ for all $x \in X \times Z$, the solutions of the closed-loop system

$$
\begin{aligned}
\dot{x} & =f(x, z)+g(x, z) u(x, z) \\
\dot{z} & =q(x, z)
\end{aligned}
$$

satisfy

$$
\|x(t)\| \leq \sqrt{\frac{c_{2}}{c_{1}}} e^{-\frac{c_{3}}{2} t}\|x(0)\| .
$$

Let $\phi_{t}(x, z)$ be the flow of (5) with initial condition $(x, z) \in X \times Z$. The flow $\phi_{t}$ is periodic with period $T>0$ and fixed point $\left(x^{*}, z^{*}\right)$ if $\phi_{T}\left(x^{*}, z^{*}\right)=\left(x^{*}, z^{*}\right)$. Associated with a periodic flow is a periodic orbit $\mathscr{O}=\left\{\phi_{t}\left(x^{*}, z^{*}\right) \in\right.$ $X \times Z: 0 \leq t \leq T\}$. Similarly, we denote the flow of the zero dynamics $\dot{z}=q(0, z)$ by $\phi_{t}^{z}$ and for a periodic flow we denote the corresponding periodic orbit by $\mathscr{O}_{Z} \subset Z$. Due to the assumption that the zero dynamics surface $Z$ is invariant, a periodic orbit for the zero dynamics, $\mathscr{O}_{Z}$, corresponds to a periodic orbit for the full-order dynamics, $\mathscr{O}=\iota_{0}\left(\mathscr{O}_{Z}\right)$, through the canonical embedding $\iota_{0}: Z \rightarrow X \times Z$ given by $\iota_{0}(z)=(0, z)$.

Without loss of generality, we can assume that the norm on $X \times Z$ is constructed from norms on $X$ and $Z$ respectively by $\|(x, z)\|=\|x\|+\|z\|$. The distance of a point $(x, z)$ from a periodic orbit $\mathscr{O}$ then satisfies

$$
\begin{aligned}
\|(x, z)\|_{\mathscr{O}} & :=\inf _{\left(x^{\prime}, z^{\prime}\right) \in \mathscr{O}}\left\|(x, z)-\left(x^{\prime}, z^{\prime}\right)\right\| \\
& =\inf _{z^{\prime} \in \mathscr{O}}\left\|z-z^{\prime}\right\|+\|x\| .
\end{aligned}
$$

A periodic orbit $\mathscr{O}$ is exponentially stable if there exist $\delta, M, \beta>0$ such that if $(x, z) \in B_{\delta}(\mathscr{O})=\{(x, z) \in$ $\left.X \times Z:\|(x, z)\|_{\mathscr{O}}<\delta\right\}$ it follows that $\left\|\phi_{t}(x, z)\right\|_{\mathscr{O}} \leq$ $M e^{-\beta t}\|(x, z)\|_{\mathscr{O}}$. The exponential stability of a periodic orbit $\mathscr{O}_{Z}$ in $Z$ can be similarly defined.

Theorem 1: (based on [16, Thm. 2, part (iv)]) For the system (1), let $\mathscr{O}_{Z}$ be an exponentially stable periodic orbit for the zero dynamics $\dot{z}=q(0, z)$. If there exists an ES-CLF $V$, then for all locally Lipschitz continuous feedbacks $u(x, z)$ taking values in $K(x, z), \mathscr{O}=\iota_{0}\left(\mathscr{O}_{Z}\right)$ is an exponentially stable periodic orbit of (5).

The proof is given in the Appendix.

Theorem 1 provides conditions under which the exponential stability of a periodic orbit of a system can be determined from two lower-dimensional problems, specifically, establishing the exponential stability of a periodic orbit contained in a zero dynamics submanifold and constructing an ES-CLF for the dynamics transverse to the submanifold. The primary goal of this paper is to establish a similar result for a class of hybrid systems, where the zero dynamics will be replaced with the hybrid zero dynamics [49]. In the hybrid context, a stronger notion of convergence is needed for the transverse dynamics because the reset map in a hybrid system may act as a persistent disturbance ${ }^{1}$ and may push solutions away from the desired orbit [25]. The need for a stronger form of convergence to counter the action of the reset map will lead to the notion of a rapidly exponentially stabilizing control Lyapunov function. Rather than directly introducing this notion, we will first motivate it by revisiting input/output linearization in the context of Lyapunov functions.

\section{INPUT-OUTPUT LINEARIZATION-A LYAPUNOV PERSPECTIVE}

\section{A. Input-Output Linearization Revisited}

To motivate later constructions, we consider an affine control system modeling a mechanical system (with configuration space $\Theta$ and (local) coordinates $\theta \in \Theta$ ) given by

$$
\left[\begin{array}{l}
\dot{\theta} \\
\ddot{\theta}
\end{array}\right]=f_{\theta}(\theta, \dot{\theta})+g_{\theta}(\theta, \dot{\theta}) u
$$

for $(\theta, \dot{\theta}) \in T \Theta$. Suppose that for (7) there is an associated output $y(\theta)$ of vector relative degree 2 on a region of interest. (See [32], [19] for a definition and more detailed exposition). This results in

$$
\ddot{y}=L_{f_{\theta}}^{2} y(\theta, \dot{\theta})+L_{g_{\theta}} L_{f_{\theta}} y(\theta, \dot{\theta}) u
$$

where $L_{g_{\theta}} L_{f_{\theta}} y(\theta, \dot{\theta})$ is the decoupling matrix, which is invertible due to the vector relative degree assumption. Application of an input-output linearizing controller of the form

$$
u(\theta, \dot{\theta})=-\left(L_{g_{\theta}} L_{f_{\theta}} y(\theta, \dot{\theta})\right)^{-1}\left(L_{f_{\theta}}^{2} y(\theta, \dot{\theta})+\frac{1}{\varepsilon^{2}} K_{P} y+\frac{1}{\varepsilon} K_{D} \dot{y}\right),
$$

where $0<\varepsilon<1$ and

$$
A:=\left[\begin{array}{cc}
0 & I \\
-K_{P} & -K_{D}
\end{array}\right]
$$

\footnotetext{
${ }^{1}$ The reader may wish to scan Sect. VII for a specific example of this phenomenon; in particular, see Fig. 3 .
} 
is Hurwitz, yields the linear system on the outputs

$$
\ddot{y}=-\frac{1}{\varepsilon^{2}} K_{P} y-\frac{1}{\varepsilon} K_{D} \dot{y} .
$$

The parameter $\varepsilon$ allows the real and imaginary parts of the roots of the characteristic equation to be scaled proportionally by $1 / \varepsilon$, and hence $0<\varepsilon<1$ has the effect of speeding up the rate of convergence while leaving the "damping ratio" unchanged.

Choosing the state variables

$$
\eta_{\varepsilon}:=\left[\begin{array}{c}
\frac{1}{\varepsilon} y \\
\dot{y}
\end{array}\right]
$$

results in

$$
\varepsilon \frac{d}{d t} \eta_{\varepsilon}(t)=A \eta_{\varepsilon}(t)
$$

and performing a change of time scale $\tau=\frac{t}{\varepsilon}$ yields

$$
\frac{d}{d \tau} \eta_{\varepsilon}(\tau)=A \eta_{\varepsilon}(\tau)
$$

Due to the Hurwitz assumption, for any $Q=Q^{T}>0$ there exists $P=P^{T}>0$ such that the Lyapunov equation is satisfied

$$
A^{T} P+P A=-Q
$$

Letting

$$
\gamma:=\frac{\lambda_{\min }(Q)}{\lambda_{\max }(P)}>0
$$

where $\lambda_{\max }(\cdot)$ and $\lambda_{\min }(\cdot)$ denote (respectively) the maximum and minimum eigenvalues of a given symmetric matrix, we apply the Rayleigh-Ritz inequality to obtain

$$
Q \geq \gamma P,
$$

that is,

$$
A^{T} P+P A+\gamma P \leq 0 .
$$

Then defining the Lyapunov function

$$
V\left(\eta_{\varepsilon}\right)=\eta_{\varepsilon}^{T} P \eta_{\varepsilon}
$$

it follows from (16) that along trajectories of (12) we have

$$
\frac{d V\left(\eta_{\varepsilon}(\tau)\right)}{d \tau} \leq-\gamma V\left(\eta_{\varepsilon}(\tau)\right)
$$

Therefore in the original time-scale (i.e. along trajectories of (11)) we have

$$
\dot{V}\left(\eta_{\varepsilon}(t)\right) \leq-\frac{\gamma}{\varepsilon} V\left(\eta_{\varepsilon}(t)\right),
$$

from which it follows that

$$
V\left(\eta_{\varepsilon}(t)\right) \leq e^{-\gamma t / \varepsilon} V\left(\eta_{\varepsilon}(0)\right) .
$$

Once again employing the Rayleigh-Ritz inequality, from (20) we obtain

$$
\left\|\eta_{\varepsilon}(t)\right\| \leq \sqrt{\frac{\lambda_{\max }(P)}{\lambda_{\min }(P)}} e^{-\frac{\gamma}{2 \varepsilon} t}\left\|\eta_{\varepsilon}(0)\right\|,
$$

which leads to

$$
\left\|\begin{array}{c}
y(t) \\
\dot{y}(t)
\end{array}\right\| \leq \frac{1}{\varepsilon} \sqrt{\frac{\lambda_{\max }(P)}{\lambda_{\min }(P)}} e^{-\frac{\gamma}{2 \varepsilon} t}\left\|\begin{array}{c}
y(0) \\
\dot{y}(0)
\end{array}\right\| .
$$

Hence, the norm of $(y(t), \dot{y}(t))$ converges to zero at an exponential rate inversely proportional to $\varepsilon$, and $(y(t), \dot{y}(t))$ can be made to converge to zero arbitrarily rapidly by choosing $\varepsilon>0$ sufficiently small.

Remark 1: In the coordinates $\eta_{\varepsilon}=[y / \varepsilon, \dot{y}]^{T}, V$ satisfies

$$
\lambda_{\min }(P)\left\|\eta_{\varepsilon}\right\|_{2}^{2} \leq V\left(\eta_{\varepsilon}\right) \leq \lambda_{\max }(P)\left\|\eta_{\varepsilon}\right\|_{2}^{2},
$$

while in the coordinates $\eta=[y, \dot{y}]^{T}$, we have

$$
\lambda_{\min }(P)\|\eta\|_{2}^{2} \leq V_{\varepsilon}(\eta) \leq \frac{1}{\varepsilon^{2}} \lambda_{\max }(P)\|\eta\|_{2}^{2},
$$

where

$$
V_{\varepsilon}(\eta)=\eta^{T}\left[\begin{array}{cc}
\frac{1}{\varepsilon} I & 0 \\
0 & I
\end{array}\right] P\left[\begin{array}{cc}
\frac{1}{\varepsilon} I & 0 \\
0 & I
\end{array}\right] \eta=: \eta^{T} P_{\varepsilon} \eta .
$$

This illustrates an important point: the Lyapunov function $V$ can be constructed in terms of a state that is dependent on $\varepsilon$, while $V$ itself is independent of $\varepsilon$, or $V$ can be viewed as a function of $\varepsilon$ with the state independent of $\varepsilon$. In what follows, we will state definitions and results in terms of the latter, i.e. the CLF will be modeled on (23).

\section{B. CLF's from Lyapunov Equations}

The convergence bounds of the previous section are based on input-output linearization. The objective here is to obtain similar bounds on convergence through control Lyapunov functions, with the specific goal of obtaining an inequality similar to (19) without having to make a specific choice of controller.

Returning to (8), suppose that a preliminary feedback controller is applied that renders the zero dynamics surface

$$
Z=\left\{(\theta, \dot{\theta}) \in T \Theta \mid y(\theta)=0, L_{f_{\theta}} y(\theta, \dot{\theta})=0\right\}
$$

invariant. An example of such a control law is given by

$$
u(\theta, \dot{\theta})=-\left(L_{g_{\theta}} L_{f_{\theta}} y(\theta, \dot{\theta})\right)^{-1} L_{f_{\theta}}^{2} y(\theta, \dot{\theta})+\mu,
$$

yielding

$$
\ddot{y}=L_{g_{\theta}} L_{f_{\theta}} y(\theta, \dot{\theta}) \mu=: \ell(y, \dot{y}, z) \mu,
$$

where $z \in Z$ represent the uncontrolled states and $\ell$ is the decoupling matrix expressed in terms of $y, \dot{y}$ and $z$.

Using the coordinates

$$
\eta=\left[\eta_{1}, \eta_{2}\right]^{T}:=[y, \dot{y}]^{T},
$$

the dynamics become

$$
\left[\begin{array}{c}
\dot{\eta}_{1} \\
\dot{\eta}_{2}
\end{array}\right]=\left[\begin{array}{c}
\eta_{2} \\
0
\end{array}\right]+\left[\begin{array}{c}
0 \\
\ell\left(\eta_{1}, \eta_{2}, z\right) \mu
\end{array}\right]
$$

and writing

$$
F=\left[\begin{array}{ll}
0 & I \\
0 & 0
\end{array}\right], \quad G=\left[\begin{array}{l}
0 \\
I
\end{array}\right],
$$

we obtain the "standard" form for a partially linearized system

$$
\dot{\eta}=F \eta+G \ell(\eta, z) \mu:=f(\eta, z)+g(\eta, z) \mu,
$$

where clearly $(F, G)$ is controllable.

We will demonstrate that, subject to the decoupling matrix being invertible, there exists a positive definite differentiable 
function $V_{\varepsilon}(\eta)$ and a constant $c>0$, such that for all $\varepsilon>0$ and for all $(\eta, z)$, there exists $\mu$ such that

$$
L_{f} V_{\varepsilon}(\eta, z)+L_{g} V_{\varepsilon}(\eta, z) \mu+\frac{c}{\varepsilon} V_{\varepsilon}(\eta) \leq 0 .
$$

In particular, let $\mu$ be given by the input-output linearization control law

$$
\mu(\eta, z)=-\ell(\eta, z)^{-1} K(\varepsilon) \eta,
$$

with $K(\varepsilon)=\left[\begin{array}{ll}\frac{1}{\varepsilon^{2}} K_{P} & \frac{1}{\varepsilon} K_{D}\end{array}\right]$ so that (30) becomes

$$
\dot{\eta}=f(\eta, z)+g(\eta, z) \mu(\eta, z)=\left[\begin{array}{cc}
0 & I \\
-\frac{1}{\varepsilon^{2}} K_{P} & -\frac{1}{\varepsilon} K_{D}
\end{array}\right] \eta
$$

which is equivalent to (11). Then defining $V_{\varepsilon}(\eta)$ as in (23) (which is equivalent to (17)), it follows from (19) that

$$
\dot{V}_{\varepsilon}(\eta, z)=L_{f} V_{\varepsilon}(\eta, z)+L_{g} V_{\varepsilon}(\eta, z) \mu(\eta, z) \leq-\frac{\gamma}{\varepsilon} V_{\varepsilon}(\eta),
$$

with

$$
\begin{aligned}
& L_{f} V_{\varepsilon}(\eta, z)=\eta^{T}\left(F^{T} P_{\varepsilon}+P_{\varepsilon} F\right) \eta, \\
& L_{g} V_{\varepsilon}(\eta, z)=2 \eta^{T} P_{\varepsilon} G \ell(\eta, z),
\end{aligned}
$$

and therefore (31) is satisfied with $c=\gamma$. More generally, this shows that

$$
\inf _{\mu}\left[L_{f} V_{\varepsilon}(\eta, z)+L_{g} V_{\varepsilon}(\eta, z) \mu+\frac{\gamma}{\varepsilon} V_{\varepsilon}(\eta)\right] \leq 0,
$$

since we have produced a specific example of $\mu$ that satisfies this condition. Therefore, $V_{\varepsilon}$ is a control Lyapunov function; moreover, it is a specific example of a stronger form of CLFa rapidly exponentially stabilizing control Lyapunov function. (A precise definition will be given in Section IV.)

While (32) is one example of a control law satisfying (31), there exist many other control laws which also satisfy the bound. For example, if we define

$$
\begin{aligned}
& \psi_{0, \varepsilon}(\eta, z)=L_{f} V_{\varepsilon}(\eta, z)+\frac{\gamma}{\varepsilon} V_{\varepsilon}(\eta) \\
& \psi_{1, \varepsilon}(\eta, z)=L_{g} V_{\varepsilon}(\eta, z)^{T}
\end{aligned}
$$

in terms of (23) and (35), then the "universal" construction of Sontag [37] yields a control law which satisfies (31), given by

$$
\mu(\eta, z)=\left\{\begin{array}{llr}
\Upsilon(\eta, z) & \text { if } & \psi_{1, \varepsilon}(\eta, z) \neq 0 \\
0 & \text { if } & \psi_{1, \varepsilon}(\eta, z)=0
\end{array}\right.
$$

where

$$
\begin{aligned}
\Upsilon(\eta, z)= & {\left[\frac{-\psi_{0, \varepsilon}(\eta, z)}{\psi_{1, \varepsilon}(\eta, z)^{T} \psi_{1, \varepsilon}(\eta, z)}\right.} \\
& \left.+\frac{\sqrt{\left(\psi_{0, \varepsilon}(\eta, z)\right)^{2}+\left(\psi_{1, \varepsilon}(\eta, z)^{T} \psi_{1, \varepsilon}(\eta, z)\right)^{2}}}{\psi_{1, \varepsilon}(\eta, z)^{T} \psi_{1, \varepsilon}(\eta, z)}\right] \psi_{1, \varepsilon}(\eta, z) .
\end{aligned}
$$

\section{RAPIDLY EXPONENTIALLY StABILIZING CLF}

Motivated by Sect. III, we return to systems of the form given in (1) and consider a particular class of control Lyapunov functions which will satisfy the type of bound given by (36).

\section{A. RES-CLF}

Def. 1 is now extended to provide a means of adjusting the rate of convergence to be "sufficiently rapid". Once again, this will be important in the case of hybrid systems for which a controller for the continuous dynamics must provide adequate contraction to overcome the repulsion of the discrete dynamics.

Definition 2: For the system (1), a one-parameter family of continuously differentiable functions $V_{\varepsilon}: X \rightarrow \mathbb{R}$ is said to be a rapidly exponentially stabilizing control Lyapunov function $(\boldsymbol{R E S}-\boldsymbol{C L F})$ if there exist positive constants $c_{1}, c_{2}, c_{3}>0$ such that for all $0<\varepsilon<1$ and for all $(x, z) \in X \times Z$,

$$
\begin{aligned}
& c_{1}\|x\|^{2} \leq V_{\varepsilon}(x) \leq \frac{c_{2}}{\varepsilon^{2}}\|x\|^{2} \\
& \inf _{u \in U}\left[L_{f} V_{\varepsilon}(x, z)+L_{g} V_{\varepsilon}(x, z) u+\frac{c_{3}}{\varepsilon} V_{\varepsilon}(x)\right] \leq 0 .
\end{aligned}
$$

In the context of RES-CLF, the set $K$ introduced in (4) becomes

$$
K_{\varepsilon}(x, z)=\left\{u \in U: L_{f} V_{\varepsilon}(x, z)+L_{g} V_{\varepsilon}(x, z) u+\frac{c_{3}}{\varepsilon} V_{\varepsilon}(x) \leq 0\right\}
$$

consisting of the control values that result in $\dot{V}_{\varepsilon}(x, z, u) \leq$ $-\frac{c_{3}}{\varepsilon} V_{\varepsilon}(x)$. Just as in Sect. III, for any Lipschitz continuous feedback control law $u_{\varepsilon}(x, z)$ with values in $K_{\varepsilon}(x, z)$, (39) and (40) imply that the solutions of the closed-loop system

$$
\begin{aligned}
\dot{x} & =f(x, z)+g(x, z) u_{\varepsilon}(x, z) \\
\dot{z} & =q(x, z),
\end{aligned}
$$

satisfy

$$
V_{\varepsilon}(x(t)) \leq e^{-\frac{c_{3}}{\varepsilon} t} V_{\varepsilon}(x(0))
$$

and

$$
\|x(t)\| \leq \frac{1}{\varepsilon} \sqrt{\frac{c_{2}}{c_{1}}} e^{-\frac{c_{3}}{2 \varepsilon} t}\|x(0)\| .
$$

The rate of exponential convergence can therefore be directly controlled with the constant $\varepsilon$ through $\frac{c_{3}}{\varepsilon}$.

\section{B. Remarks on inverse optimality}

In practical applications, it can be interesting to select the control value of minimum norm in $K_{\varepsilon}(x, z)$

$$
m(x, z)=\operatorname{argmin}\left\{\|u\|: u \in K_{\varepsilon}(x, z)\right\},
$$

termed the pointwise min-norm control law [10]. For a system of the form (1) with $V_{\varepsilon}$ a RES-CLF, define

$$
\begin{aligned}
& \psi_{0, \varepsilon}(x, z)=L_{f} V_{\varepsilon}(x, z)+\frac{c_{3}}{\varepsilon} V_{\varepsilon}(x) \\
& \psi_{1, \varepsilon}(x, z)=L_{g} V_{\varepsilon}(x, z)^{T} .
\end{aligned}
$$

In this case, the pointwise min-norm control law is given by

$$
m_{\varepsilon}(x, z)=\left\{\begin{array}{lll}
-\frac{\psi_{0, \varepsilon}(x, z) \psi_{1, \varepsilon}(x, z)}{\psi_{1, \varepsilon}(x, z)^{T} \psi_{1, \varepsilon}(x, z)} & \text { if } & \psi_{0, \varepsilon}(x, z)>0 \\
0 & \text { if } & \psi_{0, \varepsilon}(x, z) \leq 0,
\end{array}\right.
$$

and is locally Lipschitz continuous.

In the previous section, a specific example of a RES-CLF was given by (23), with $c_{1}=\lambda_{\min }(P), c_{2}=\lambda_{\max }(P)$, and 
$c_{3}=\gamma$. Picking $\mu_{\varepsilon}(\eta, z)=m_{\varepsilon}(\eta, z)$, with $\psi_{0, \varepsilon}$ and $\psi_{1, \varepsilon}$ defined in (37), results in the pointwise min-norm control law such that (31) is satisfied.

It is important to note (see [10]) that the pointwise minnorm control law is actually optimal with respect to some cost function

$$
J(x, z, u)=\int_{0}^{\infty} L(x, z, u) d t
$$

and hence it exponentially stabilizes $x$ to 0 in an "optimal" manner, although the functional $L$ can be difficult to compute in closed form and may or may not be physically meaningful for a given choice of $V_{\varepsilon}$. With an eye toward optimality, the algebraic Riccati equation can be used to construct a RESCLF when the transverse dynamics is feedback linearizable, as in (26). For example, let $F$ and $G$ be defined as in (29) and let $P$ be the solution to the Riccati equation

$$
F^{T} P+P F-P G G^{T} P+Q=0
$$

for some $Q=Q^{T}>0$. It again follows that $\gamma P \leq Q$, where $\gamma$ is defined as in (14), and letting $P_{\varepsilon}=\left[\begin{array}{cc}\frac{1}{\varepsilon} I & 0 \\ 0 & I\end{array}\right] P\left[\begin{array}{cc}\frac{1}{\varepsilon} I & 0 \\ 0 & I\end{array}\right]=$ : $M_{\varepsilon} P M_{\varepsilon}$ as in (23), one can show that (46) implies

$$
F^{T} P_{\varepsilon}+P_{\varepsilon} F-\frac{1}{\varepsilon} P_{\varepsilon} G G^{T} P_{\varepsilon}+\frac{1}{\varepsilon} M_{\varepsilon} Q M_{\varepsilon}=0 .
$$

Then defining $\eta$ as in (27) with dynamics (30) and letting $V_{\varepsilon}(\eta)=\eta^{T} P_{\varepsilon} \eta$, we have

$$
\dot{V}_{\varepsilon}(\eta, z, \mu)+\frac{\gamma}{\varepsilon} V_{\varepsilon}(\eta) \leq \eta^{T} P_{\varepsilon} G\left(\frac{1}{\varepsilon} G^{T} P_{\varepsilon}+2 \ell(\eta, z) \mu\right),
$$

from which it follows that

$$
\inf _{\mu \in U}\left[\dot{V}_{\varepsilon}(\eta, z, \mu)+\frac{\gamma}{\varepsilon} V_{\varepsilon}(\eta)\right] \leq 0 .
$$

$V_{\varepsilon}\left(\eta_{\varepsilon}\right)$ is therefore a RES-CLF with $c_{1}=\lambda_{\min }(P), c_{2}=$ $\lambda_{\max }(P)$, and $c_{3}=\gamma$, and for any feedback control law $\mu_{\varepsilon}(\eta, z)$ taking values in $K_{\varepsilon}(\eta, z)$, we obtain the bounds on $\|\eta(t)\|$ given in (22). Note that the pointwise min-norm control law $\mu_{\varepsilon}(\eta, z)=m_{\varepsilon}(\eta, z)$ in (45) is a specific example of such a control law, where in this case $\psi_{0, \varepsilon}$ and $\psi_{1, \varepsilon}$ are exactly as in (44) except $P$ is now the solution of (46).

\section{CLF'S AND HYBRID SYSTEMS}

This section considers control Lyapunov functions in the context of hybrid systems. Analogous to the case of non-hybrid systems in (1), we will begin with the assumption that the hybrid system already has a hybrid zero dynamics ([47], [25]), which is a manifold that is invariant under both the continuous and the discrete dynamics of the hybrid system. The goal is to provide conditions for establishing the local exponential stability of a periodic orbit of a hybrid system on the basis of two lower-dimensional problems, namely, establishing the exponential stability of a periodic orbit contained in the hybrid zero dynamics and constructing a RES-CLF for the dynamics transverse to the submanifold.

\section{A. Hybrid systems and zero dynamics}

Consider a hybrid control system of the form of a system with impulse effects [6], [50], [17]

$$
\mathscr{H} \mathscr{C}=\left\{\begin{array}{rlrl}
\dot{x} & =f(x, z)+g(x, z) u & \text { if } \quad & (x, z) \in D \backslash S \\
\dot{z} & =q(x, z) & & \\
x^{+} & =\Delta_{X}\left(x^{-}, z^{-}\right) \\
z^{+} & =\Delta_{Z}\left(x^{-}, z^{-}\right)
\end{array} \quad \text { if } \quad\left(x^{-}, z^{-}\right) \in S\right.
$$

where $x \in X, z \in Z, u \in U$ are defined as in (1), the domain $D$ is a closed subset of $X \times Z$, the functions $f, g, q, \Delta_{X}, \Delta_{Z}$ are locally Lipschitz in their arguments, and the guard or switching surface $S \subset D$ is a co-dimension one submanifold of $D$. We assume furthermore that the domain and switching surfaces are given by

$$
\begin{aligned}
D & =\{(x, z) \in X \times Z: h(x, z) \geq 0\} \\
S & =\{(x, z) \in X \times Z: h(x, z)=0 \text { and } \dot{h}(x, z)<0\},
\end{aligned}
$$

for some continuously differentiable function $h: X \times Z \rightarrow \mathbb{R}$ for which $L_{g} h=0$. For simplicity of notation, we write $\Delta(x, z)=\left(\Delta_{X}(x, z), \Delta_{Z}(x, z)\right)$ which is the reset map representing the discrete dynamics of the hybrid system.

As previously mentioned, it is assumed that $\mathscr{H} \mathscr{C}$ has a hybrid zero dynamics. More specifically, we assume that $f(0, z)=0$, so that the surface $Z$ is invariant for the continuous dynamics, and that $\Delta_{X}(0, z)=0$, so that the surface $Z$ is invariant for the discrete dynamics. The hybrid zero dynamics is then the hybrid system

$$
\left.\mathscr{H}\right|_{Z}=\left\{\begin{array}{rlrr}
\dot{z} & =q(0, z) & \text { if } & z \in Z \backslash(S \cap Z) \\
z^{+} & =\Delta_{Z}\left(0, z^{-}\right) & \text {if } & z^{-} \in S \cap Z .
\end{array}\right.
$$

For a hybrid system of the form (49) and a RES-CLF $V_{\varepsilon}(x)$, we can again consider locally Lipschitz continuous control laws $u_{\varepsilon}(x, z) \in K_{\varepsilon}(x, z)$. Applying such a control results in the closed-loop hybrid system

$$
\mathscr{H}_{\varepsilon}=\left\{\begin{array}{rlrl}
\dot{x} & =f(x, z)+g(x, z) u_{\varepsilon}(x, z) & & \text { if } \quad \\
\dot{z} & =q(x, z) \in D \backslash S \\
x^{+} & =\Delta\left(x^{-}, z^{-}\right) & \text {if } \quad & \left(x^{-}, z^{-}\right) \in S \\
z^{+} & &
\end{array}\right.
$$

Because $u_{\varepsilon}(x, z) \in K_{\varepsilon}(x, z)$ implies $u_{\varepsilon}(0, z)=0$, the hybrid zero dynamics $\left.\mathscr{H}\right|_{Z}$ is preserved.

\section{B. Solutions, periodic orbits, and the Poincaré map}

In the context of hybrid systems, one can define solutions in many ways [50], [17], [49], [12]. Because we focus on periodic orbits and solutions that evolve in a neighborhood of such orbits, we are primarily interested in the Poincaré map, which can be defined rather easily for the hybrid systems considered here. Therefore, in the interest of brevity and simplicity, we will forgo a full description of solutions of hybrid systems.

For the hybrid system $\mathscr{H}_{\varepsilon}$, let $\phi_{t}^{\varepsilon}(x, z)$ be a flow of the continuous dynamics of (52) (i.e. the dynamics given by (41)). For $\left(x^{*}, z^{*}\right) \in S$, we say that $\phi_{t}^{\varepsilon}$ is hybrid periodic with period $T>0$ if $\phi_{T}^{\varepsilon}\left(\Delta\left(x^{*}, z^{*}\right)\right)=\left(x^{*}, z^{*}\right)$. (Note that here we are assuming that the fixed point is in the switching surface $S$; more general definitions are possible [14], [46], but the one 


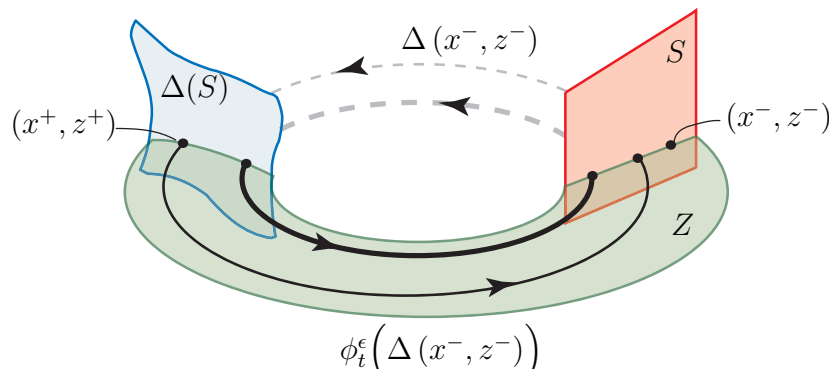

Fig. 1: Trajectories of the hybrid system (52) lying in the zero dynamics, where $Z$ is the zero dynamics surface and $S$ is the switching surface (50). The reset map $\Delta$ maps an initial point $\left(x^{-}, z^{-}\right) \in S$ to $\left(x^{+}, z^{+}\right) \in \Delta(S)$, which then serves as the initial condition for $\phi_{t}^{\varepsilon}$, the flow of the continuous dynamics in (49). The thicker trajectory denotes a hybrid periodic orbit lying in $Z$.

introduced is sufficient for our purposes.) A set $\mathscr{O}$ is a hybrid periodic orbit if $\mathscr{O}=\left\{\phi_{t}^{\varepsilon}\left(\Delta\left(x^{*}, z^{*}\right)\right): 0 \leq t \leq T\right\}$ for a hybrid periodic flow $\phi_{t}^{\varepsilon}$. Figure 1 depicts two solutions lying in the zero dynamics manifold, one of which is periodic.

Taking $S$ as a Poincaré section, associated with a hybrid periodic orbit is a Poincaré map $P^{\varepsilon}: S \rightarrow S$ which is a partial function

$$
P^{\varepsilon}(x, z)=\phi_{T_{I}^{\varepsilon}(x, z)}^{\varepsilon}(\Delta(x, z)),
$$

where $T_{I}^{\varepsilon}: S \rightarrow D$ is the time-to-impact function defined by

$$
T_{I}^{\varepsilon}(x, z)=\inf \left\{t \geq 0: \phi_{t}^{\varepsilon}(\Delta(x, z)) \in S\right\}
$$

and obtained through the implicit function theorem by considering the function $H(t, x, z)=h\left(\phi_{t}^{\varepsilon}(\Delta(x, z))\right)$ for which $H\left(T, x^{*}, z^{*}\right)=0$. Since, by assumption on $S$, $\frac{\partial H}{\partial t}\left(T, x^{*}, z^{*}\right)<0$, the implicit function theorem implies that $T_{I}^{\varepsilon}$ is well-defined in a neighborhood of $\left(x^{*}, z^{*}\right)$. Therefore, $T_{I}^{\varepsilon}\left(x^{*}, z^{*}\right)=T$ and so $P^{\varepsilon}\left(x^{*}, z^{*}\right)=\left(x^{*}, z^{*}\right)$. Also, we note that $H(t, x, z)$ is Lipschitz continuous since it is differentiable in $t, h$ is assumed to be continuously differentiable, and $\phi_{t}^{\varepsilon}(\Delta(x, z))$ is Lipschitz continuous, and therefore $T_{I}^{\varepsilon}$ is also Lipschitz [44].

A hybrid periodic orbit, $\mathscr{O}_{Z}$, of $\left.\mathscr{H}\right|_{Z}$ can be similarly defined, in which case the corresponding Poincaré map $\rho$ : $S \cap Z \rightarrow S \cap Z$ (which is again a partial function) is termed the restricted Poincaré map [26]. In this case,

$$
\rho(z)=\phi_{T_{\rho}(z)}^{z}\left(\Delta_{Z}(0, z)\right),
$$

where $\phi^{z}$ is the flow of $\dot{z}=q(0, z)$ and $T_{\rho}(z)$ is the restricted time-to-impact function which is simply given by $T_{\rho}(z):=T_{I}^{\varepsilon}(0, z)$. Due to the assumption that the zero dynamics surface is invariant, a periodic orbit for the zero dynamics, $\mathscr{O}_{Z}$, corresponds to a periodic orbit for the fullorder dynamics, $\mathscr{O}=\iota_{0}\left(\mathscr{O}_{Z}\right)$, through the canonical embedding $\iota_{0}: Z \rightarrow X \times Z$ given by $\iota_{0}(z)=(0, z)$. It follows that we can assume that $x^{*}=0$. Moreover, without loss of generality, we assume that $z^{*}=0$ as well.

As with non-hybrid dynamical systems, the stability of the Poincare map determines the stability of the hybrid periodic orbit $\mathscr{O}$. Specifically, the Poincaré map is (locally) exponentially stable (as a discrete time system with $\left(x_{k+1}, z_{k+1}\right)=$ $\left.P^{\varepsilon}\left(x_{k}, z_{k}\right)\right)$ at the fixed point $\left(x^{*}, z^{*}\right)$ if and only if the hybrid periodic orbit $\mathscr{O}$ is exponentially stable ${ }^{2}$ [26]. It is this fact that we will utilize in proving the main result of the paper. Similarly, the stability of a periodic orbit $\mathscr{O}_{Z}$ in the hybrid zero dynamics is determined by the restricted Poincaré map.

\section{Main result}

With the notation of the previous section in hand, we now present the main result of this paper.

Theorem 2: Let $\mathscr{O}_{Z}$ be an exponentially stable periodic orbit of the hybrid zero dynamics $\left.\mathscr{H}\right|_{Z}$ transverse to $S \cap Z$ and assume there exists a RES-CLF $V_{\varepsilon}$ for the continuous dynamics (1) of $\mathscr{H} \mathscr{C}$. Then there exists an $\bar{\varepsilon}>0$ such that for all $0<\varepsilon<\bar{\varepsilon}$ and for all Lipschitz continuous $u_{\varepsilon}(x, z) \in K_{\varepsilon}(x, z), \mathscr{O}=\iota_{0}\left(\mathscr{O}_{Z}\right)$ is an exponentially stable hybrid periodic orbit of $\mathscr{H}_{\varepsilon}$.

\section{PROOF OF MAIN RESULT}

Using the equivalency of the exponential stability of a hybrid periodic orbit and the exponential stability of the corresponding fixed point of the Poincare map, the proof seeks a Lyapunov function $V_{P_{\varepsilon}}$, defined locally about $\left(x^{*}, z^{*}\right)=$ $(0,0) \in S$, for the Poincaré map $P^{\varepsilon}$; that is, it seeks a (local) Lyapunov function for the discrete-time system $\left(x_{k+1}, z_{k+1}\right)=P^{\varepsilon}\left(x_{k}, z_{k}\right)$ with equilibrium point $(0,0)$. Roughly speaking, $\left.V_{P_{\varepsilon}}\right|_{S \cap Z}$ will be constructed on the basis of the restricted Poincaré map $\rho: S \cap Z \rightarrow S \cap Z$ with $\rho\left(z^{*}\right)=z^{*}$, while the "transversal" component will be constructed from the RES-CLF $V_{\varepsilon}$ restricted to $S$.

Before proving Theorem 2, we first state and prove a lemma establishing a bound on the Poincaré map in terms of the restricted Poincaré map and a bound on the time-to-impact function $T_{I}^{\varepsilon}(x, z)$ in terms of $T_{\rho}(z)$. In the following, $B_{\delta}(r)$ denotes an open ball of radius $\delta>0$ centered on the point $r$, and $P_{z}^{\varepsilon}(x, z)$ is the z-component of $P^{\varepsilon}(x, z)$.

Lemma 1: Let $\mathscr{O}_{Z}$ be a periodic orbit of the hybrid zero dynamics $\left.\mathscr{H}\right|_{Z}$ transverse to $S \cap Z$ and assume there exists a RES-CLF $V_{\varepsilon}$ for the continuous dynamics (1) of $\mathscr{H} \mathscr{C}$. Then there exist finite constants $L_{T_{I}}$ and $A_{1}$ (both independent of $\varepsilon)$ such that for all $\varepsilon>0$ and for all Lipschitz continuous $u_{\varepsilon}(x, z) \in K_{\varepsilon}(x, z)$ there exists $a \delta>0$ such that for all $(x, z) \in B_{\delta}(0,0) \cap S$,

$$
\begin{aligned}
\left\|T_{I}^{\varepsilon}(x, z)-T_{\rho}(z)\right\| & \leq L_{T_{I}}\|x\|, \\
\left\|P_{z}^{\varepsilon}(x, z)-\rho(z)\right\| & \leq A_{1}\|x\| .
\end{aligned}
$$

Proof: In the first step of the proof, we construct an auxiliary time-to-impact function $T_{B}$ that is Lipschitz continuous and independent of $\varepsilon$ and then relate it to $T_{I}^{\varepsilon}$.

Recall that $h(x, z)$ is the guard. Let $\mu_{1} \in \mathbb{R}^{n_{x}}$ and $\mu_{2} \in \mathbb{R}^{n_{z}}$ be constant vectors and let $\phi_{t}^{z}\left(\Delta\left(0, z_{0}\right)\right)$ be the solution of

\footnotetext{
${ }^{2}$ While Theorem 1 in [26] assumes the hybrid model is $C^{1}$, its proof only requires that $f$ and $\Delta$ are Lipschitz continuous.
} 
$\dot{z}=q(0, z)$ with $z(0)=\Delta_{Z}\left(0, z_{0}\right)$. Define

$$
T_{B}\left(\mu_{1}, \mu_{2}, z\right)=\inf \left\{t \geq 0: h\left(\mu_{1}, \phi_{t}^{z}(\Delta(0, z))+\mu_{2}\right)=0\right\},
$$

wherein it follows that $T_{B}(0,0, z)=T_{\rho}(z)$. By construction, $T_{B}$ is independent of $\varepsilon$ and (by the same argument used for $\left.T_{I}^{\varepsilon}(x, z)\right)$ is Lipschitz continuous. Hence, in the norm $\left\|\left(\mu_{1}, \mu_{2}, z\right)\right\|:=\left\|\mu_{1}\right\|+\left\|\mu_{2}\right\|+\|z\|$,

$$
\left|T_{B}\left(\mu_{1}, \mu_{2}, z\right)-T_{\rho}(z)\right| \leq L_{B}\left(\left\|\mu_{1}\right\|+\left\|\mu_{2}\right\|\right),
$$

where $L_{B}$ is the (local) Lipschitz constant.

Let $\varepsilon>0$ be fixed and select a Lipschitz continuous feedback $u_{\varepsilon} \in K_{\varepsilon}(x, z)$. We note that $T_{I}^{\varepsilon}(x, z)$ is continuous (since it is Lipschitz) and therefore there exists $\delta>0$ such that for all $(x, z) \in B_{\delta}(0,0) \cap S$

$$
0.9 T^{*} \leq T_{I}^{\varepsilon}(x, z) \leq 1.1 T^{*},
$$

where $T^{*}=T_{\rho}(0)$ is the period of the orbit $\mathscr{O}_{Z}$. Let $\left(x_{1}(t), z_{1}(t)\right)$ satisfy $\dot{z}_{1}(t)=q\left(x_{1}(t), z_{1}(t)\right)$ with $x_{1}(0)=$ $\Delta_{X}(x, z)$ and $z_{1}(0)=\Delta_{Z}(x, z)$, and similarly, let $z_{2}(t)$ satisfy $\dot{z}_{2}(t)=q\left(0, z_{2}(t)\right)$ with $z_{2}(0)=\Delta_{Z}(0, z)$.

Defining

$$
\begin{aligned}
& \mu_{1}=\left.x_{1}(t)\right|_{t=T_{I}^{\varepsilon}(x, z)} \\
& \mu_{2}=\left.z_{1}(t)\right|_{t=T_{I}^{\varepsilon}(x, z)}-\left.z_{2}(t)\right|_{t=T_{I}^{\varepsilon}(x, z)},
\end{aligned}
$$

results in

$$
T_{B}\left(\mu_{1}, \mu_{2}, z\right)=T_{I}^{\varepsilon}(x, z)
$$

because $T_{I}^{\varepsilon}$ and $T_{B}$ are locally unique solutions where the guard vanishes (follows from Implicit Function Theorem). We will establish (53) by bounding $\mu_{1}$ and $\mu_{2}$ and substituting into (55) by virtue of (58), as follows.

Using the fact that $V_{\varepsilon}$ is rapidly exponentially stabilizing, we have the bound from (43) given by

$$
\left\|x_{1}(t)\right\| \leq \sqrt{\frac{c_{2}}{c_{1}}} \frac{1}{\varepsilon} e^{-\frac{c_{3}}{2 \varepsilon} t}\left\|x_{1}(0)\right\|
$$

Note that $\Delta_{X}(0, z)=0$ and therefore $\left\|x_{1}(0)\right\|=\| \Delta_{X}(x, z)-$ $\Delta_{X}(0, z)\left\|\leq L_{\Delta_{X}}\right\| x \|$. Then making use of (56), we have

$$
\begin{aligned}
\left\|\mu_{1}\right\| & =\left\|x_{1}(t)\right\|_{t=T_{I}^{\varepsilon}(x, z)} \\
& \leq \sqrt{\frac{c_{2}}{c_{1}}} \frac{1}{\varepsilon} e^{-\frac{c_{3}}{2 \varepsilon} 0.9 T^{*}} L_{\Delta_{X}}\|x\| \\
& \leq \frac{2 e^{-1}}{0.9 T^{*} c_{3}} \sqrt{\frac{c_{2}}{c_{1}}} L_{\Delta_{X}}\|x\| .
\end{aligned}
$$

The next step is to bound $\left\|\mu_{2}\right\|$ using a Gronwall-Bellman argument. We first note that

$$
z_{1}(t)-z_{2}(t)=z_{1}(0)-z_{2}(0)+\int_{0}^{t} q\left(x_{1}(\tau), z_{1}(\tau)\right)-q\left(0, z_{2}(\tau)\right) d \tau
$$

and thus

$$
\begin{aligned}
\left\|z_{1}(t)-z_{2}(t)\right\| \leq & L_{\Delta_{Z}}\|x\|+\int_{0}^{t} L_{q}\left(\left\|x_{1}(\tau)\right\|+\left\|z_{1}(\tau)-z_{2}(\tau)\right\|\right) d \tau \\
\leq & L_{\Delta_{Z}}\|x\|+\frac{2}{c_{3}} \sqrt{\frac{c_{2}}{c_{1}}} L_{q} L_{\Delta_{X}}\|x\| \\
& \quad+\int_{0}^{t} L_{q}\left(\left\|z_{1}(\tau)-z_{2}(\tau)\right\|\right) d \tau
\end{aligned}
$$

where (59) has been substituted, integrated, and bounded. Hence, by the Gronwall-Bellman inequality,

$$
\left\|z_{1}(t)-z_{2}(t)\right\| \leq\left(L_{\Delta_{Z}}+\frac{2}{c_{3}} \sqrt{\frac{c_{2}}{c_{1}}} L_{q} L_{\Delta_{X}}\right)\|x\| e^{L_{q} t},
$$

and therefore $\left\|\mu_{2}\right\| \leq C_{1} e^{1.1 L_{q} T^{*}}\|x\|$, where $C_{1}$ is the term in parentheses in (60). The proof of (53) is then completed by substituting the bounds for $\left\|\mu_{1}\right\|$ and $\left\|\mu_{2}\right\|$ into (55) and grouping terms.

To establish (54), we first define

$$
C_{2}=\max _{.9 T^{*} \leq t \leq 1.1 T^{*}}\left\|q\left(0, z_{2}(t)\right)\right\| .
$$

It then follows from (53), (56) and (60) that

$$
\begin{aligned}
\| P_{z}^{\varepsilon}(x, z)- & \rho(z) \| \\
\leq & \left\|z_{1}(0)-z_{2}(0)\right\| \\
& +\int_{0}^{T_{I}^{\varepsilon}(x, z)}\left\|q\left(x_{1}(\tau), z_{1}(\tau)\right)-q\left(0, z_{2}(\tau)\right)\right\| d \tau \\
& +\left|\int_{T_{I}^{\varepsilon}(x, z)}^{T_{\rho}(z)}\left\|q\left(0, z_{2}(\tau)\right)\right\| d \tau\right| \\
\leq & \left(C_{1} e^{1.1 L_{q} T^{*}}+C_{2} L_{T_{I}}\right)\|x\|
\end{aligned}
$$

which establishes (54).

We now have the necessary framework in which to prove Theorem 2.

Proof: [of Theorem 2] The results of Lemma 1 and the exponential stability of $\mathscr{O}_{Z}$ imply that there exists a $\delta>0$ such that $\rho: B_{\delta}(0) \cap(S \cap Z) \rightarrow B_{\delta}(0) \cap(S \cap Z) \rightarrow$ is well-defined for all $z \in B_{\delta}(0) \cap(S \cap Z)$ and $z_{k+1}=\rho\left(z_{k}\right)$ is (locally) exponentially stable, i.e., $\left\|z_{k}\right\| \leq N \alpha^{k}\left\|z_{0}\right\|$ for some $N>0,0<\alpha<1$ and all $k \geq 0$. Therefore, by the converse Lyapunov theorem for discrete-time systems, there exists a Lyapunov function $V_{\rho}$, defined on $B_{\delta}(0) \cap(S \cap Z)$ for some $\delta>0$ (possibly smaller than the previously defined $\delta$ ), and positive constants $r_{1}, r_{2}, r_{3}, r_{4}$ satisfying

$$
\begin{gathered}
r_{1}\|z\|^{2} \leq V_{\rho}(z) \leq r_{2}\|z\|^{2} \\
V_{\rho}(\rho(z))-V_{\rho}(z) \leq-r_{3}\|z\|^{2} \\
\left|V_{\rho}(z)-V_{\rho}\left(z^{\prime}\right)\right| \leq r_{4}\left\|z-z^{\prime}\right\|\left(\|z\|+\left\|z^{\prime}\right\|\right) .
\end{gathered}
$$

For the RES-CLF $V_{\varepsilon}$, denote its restriction to the switching surface $S$ by $V_{\varepsilon, X}=\left.V_{\varepsilon}\right|_{S}$. With these two Lyapunov functions (motivated by the construction from [23] for singularly perturbed systems) we define the following candidate Lyapunov function

$$
V_{P_{\varepsilon}}(x, z)=V_{\rho}(z)+\sigma V_{\varepsilon, X}(x)
$$

defined on $B_{\delta}(0,0) \subset S$, where $\sigma>0$ is any constant such that $\sigma>\bar{\sigma}>0$. (We will define $\bar{\sigma}$ explicitly later.) By (39) and (61), it is clear that

$\min \left\{\sigma c_{1}, r_{1}\right\}\|(x, z)\|^{2} \leq V_{P_{\varepsilon}}(x, z) \leq \max \left\{\sigma \frac{c_{2}}{\varepsilon^{2}}, r_{2}\right\}\|(x, z)\|^{2}$.

Noting that $\|(x, z)\|^{2}=\|x\|^{2}+\|z\|^{2}+2\|x\|\|z\| \geq\|x\|^{2}+$ $\|z\|^{2}$, in order to establish exponential stability of the origin 
for the discrete-time system $\left(x_{k+1}, z_{k+1}\right)=P^{\varepsilon}\left(x_{k}, z_{k}\right)$, it is sufficient to establish that

$$
V_{P_{\varepsilon}}\left(P^{\varepsilon}(x, z)\right)-V_{P_{\varepsilon}}(x, z) \leq-\kappa\left(\|x\|^{2}+\|z\|^{2}\right),
$$

for some $\kappa>0$. Since $P^{\varepsilon}(x, z) \in S \subset X \times Z$, we denote the $X$ and $Z$ components of $P^{\varepsilon}$ by $P_{x}^{\varepsilon}(x, z)$ and $P_{z}^{\varepsilon}(x, z)$, respectively. With this notation,

$$
\begin{aligned}
V_{P_{\varepsilon}}\left(P^{\varepsilon}(x, z)\right)-V_{P_{\varepsilon}}(x, z) & =V_{\rho}\left(P_{z}^{\varepsilon}(x, z)\right)-V_{\rho}(z) \\
& +\sigma\left(V_{\varepsilon, X}\left(P_{x}^{\varepsilon}(x, z)\right)-V_{\varepsilon, X}(x)\right) .
\end{aligned}
$$

We begin by noting that, because $V_{\varepsilon}$ is a RESCLF and $u_{\varepsilon}(x, z) \in K_{\varepsilon}(x, z)$, and since $P_{x}^{\varepsilon}(x, z)=$ $\left(\phi_{T_{I}^{\varepsilon}(x, z)}^{\varepsilon}(\Delta(x, z))\right)_{x}$, it follows from (39) and (42) that

$$
\begin{aligned}
V_{\varepsilon, X}\left(P_{x}^{\varepsilon}(x, z)\right) & \leq \frac{c_{2}}{\varepsilon^{2}} e^{-\frac{c_{3}}{\varepsilon} T_{I}^{\varepsilon}(x, z)}\left\|\Delta_{X}(x, z)\right\|^{2} \\
& \leq \frac{c_{2}}{\varepsilon^{2}} L_{\Delta_{X}}^{2} e^{-\frac{c_{3}}{\varepsilon} T_{I}^{\varepsilon}(x, z)}\|x\|^{2},
\end{aligned}
$$

where the last inequality follows from the fact that $\Delta_{X}(0, z)=$ 0 and therefore

$$
\left\|\Delta_{X}(x, z)\right\|^{2}=\left\|\Delta_{X}(x, z)-\Delta_{X}(0, z)\right\|^{2} \leq L_{\Delta_{X}}^{2}\|x\|^{2},
$$

with $L_{\Delta_{X}}$ the Lipschitz constant for $\Delta_{X}$. Defining $\beta_{1}(\varepsilon)=$ $\frac{c_{2}}{\varepsilon^{2}} L_{\Delta X}^{2} e^{-\frac{c_{3}}{\varepsilon} \cdot 9 T^{*}}$ (with $T^{*}$ defined as in the proof of Lemma 1), we have established that

$$
\sigma\left(V_{\varepsilon, X}\left(P_{x}^{\varepsilon}(x, z)\right)-V_{\varepsilon, X}(x)\right) \leq \sigma\left(\beta_{1}(\varepsilon)-c_{1}\right)\|x\|^{2},
$$

where, clearly, $\beta_{1}\left(0^{+}\right):=\lim _{\varepsilon \backslash 0} \beta_{1}(\varepsilon)=0$. Therefore, there exists an $\bar{\varepsilon}$ such that

$$
\beta_{1}(\varepsilon)<c_{1} \quad \forall \quad 0<\varepsilon<\bar{\varepsilon} .
$$

As a result of Lemma 1 and the assumption that the origin is an exponentially stable equilibrium for $z_{k+1}=\rho\left(z_{k}\right)$, we have the following inequalities:

$$
\begin{aligned}
& \left\|P_{z}^{\varepsilon}(x, z)-\rho(z)\right\| \leq A_{1}\|x\|, \\
& \left\|P_{z}^{\varepsilon}(x, z)\right\|=\left\|P_{z}^{\varepsilon}(x, z)-\rho(z)+\rho(z)-\rho(0)\right\| \\
& \quad \leq A_{1}\|x\|+L_{\rho}\|z\|, \\
& \|\rho(z)\| \leq N \alpha\|z\|,
\end{aligned}
$$

where $L_{\rho}$ is the Lipschitz constant for $\rho$. Thus, using (61),

$$
\begin{aligned}
V_{\rho}\left(P_{z}^{\varepsilon}(x, z)\right)-V_{\rho}(\rho(z)) \leq & r_{4} A_{1}^{2}\|x\|^{2} \\
& +r_{4} A_{1}\left(L_{\rho}+N \alpha\right)\|x\|\|z\| .
\end{aligned}
$$

Setting $\beta_{2}=r_{4} A_{1}^{2}$ and $\beta_{3}=r_{4} A_{1}\left(L_{\rho}+N \alpha\right)$ for notational simplicity, it follows that

$$
\begin{aligned}
V_{\rho}\left(P_{z}^{\varepsilon}(x, z)\right)-V_{\rho}(z)= & V_{\rho}\left(P_{z}^{\varepsilon}(x, z)\right)-V_{\rho}(\rho(z)) \\
& +V_{\rho}(\rho(z))-V_{\rho}(z) \\
\leq & \beta_{2}\|x\|^{2}+\beta_{3}\|x\|\|z\|-r_{3}\|z\|^{2} .
\end{aligned}
$$

Therefore, combining (63), (65), and (67), we have

$$
\begin{aligned}
V_{P_{\varepsilon}}\left(P^{\varepsilon}(x, z)\right)-V_{P_{\varepsilon}}(x, z) \leq & \left(\beta_{2}+\sigma\left(\beta_{1}(\varepsilon)-c_{1}\right)\right)\|x\|^{2} \\
& +\beta_{3}\|x\|\|z\|-r_{3}\|z\|^{2} \\
= & -\left[\begin{array}{ll}
\|z\| & \|x\|
\end{array}\right] \Lambda(\varepsilon)\left[\begin{array}{l}
\|z\| \\
\|x\|
\end{array}\right],
\end{aligned}
$$

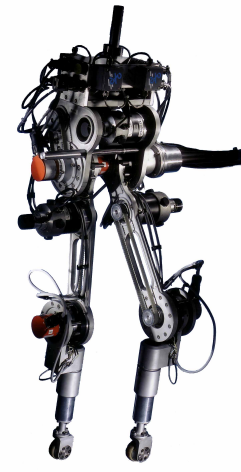

(a) RABBIT

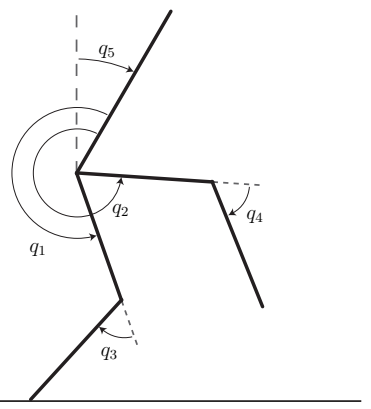

(b) Coordinate system
Fig. 2: RABBIT, a five-link bipedal walker, with the associated coordinate model. The simulation results presented in section VII are based on RABBIT's model dynamics.

with

$$
\Lambda(\varepsilon)=\left[\begin{array}{cc}
r_{3} & -\frac{1}{2} \beta_{3} \\
-\frac{1}{2} \beta_{3} & \sigma\left(c_{1}-\beta_{1}(\varepsilon)\right)-\beta_{2}
\end{array}\right] .
$$

We proceed by choosing $\bar{\sigma}>0$ such that for $\sigma>\bar{\sigma}$ and for $\varepsilon>0$ sufficiently small, $\Lambda(\varepsilon)$ is positive definite (i.e. $\operatorname{det}(\Lambda(\varepsilon))>0)$. Noting that

$$
\operatorname{det}(\Lambda(\varepsilon))=\sigma r_{3}\left(c_{1}-\beta_{1}(\varepsilon)\right)-r_{3} \beta_{2}-\frac{\beta_{3}^{2}}{4},
$$

we choose

$$
\bar{\sigma}:=\frac{4 r_{3} \beta_{2}+\beta_{3}^{2}}{4 r_{3}\left(c_{1}-\beta_{1}(\varepsilon)\right)},
$$

and by (66) we have $\bar{\sigma}>0$ for all $0<\varepsilon<\bar{\varepsilon}$. Then choosing $\kappa$ in (62) as $\kappa=\lambda_{\min }(\Lambda(\varepsilon))$, the largest eigenvalue of $\Lambda(\varepsilon)$, establishes the local exponential stability of $\mathscr{O}$.

\section{Application to Bipedal WALKing}

In this section we apply the main result of this paper in simulation with the model of a five-link bipedal robot which has a torso and two legs with revolute knees. The particular model we use is based on RABBIT (see Fig. 2), a robotic testbed developed at Laboratoire D'Automatique de Grenoble to explore fundamental issues in dynamic walking [48] and running [27]. RABBIT has four actuators to control hip and knee angles, and is connected to a rotating boom which constrains the robot to walk in a circle, approximating planar motion in the saggital plane. MATLAB code for generating the equations of motion for RABBIT is available at http://web.eecs.umich.edu/ grizzle/biped_book_web/.

Detailed descriptions of RABBIT and the associated mathematical model can be found in [9], [48]; we briefly summarize the model construction as follows. In the stance phase, a suitable set of coordinates is given by $q:=\left(q_{1}, q_{2}, q_{3}, q_{4}, q_{5}\right)$ as illustrated in Fig. 2, where $q_{1}$ and $q_{2}$ are the femur angles (referenced to the torso), $q_{3}$ and $q_{4}$ are the knee angles, and $q_{5}$ is the absolute angle of the torso. The method of Lagrange yields the standard second order system

$$
D(q) \ddot{q}+C(q, \dot{q}) \dot{q}+G(q)=B(q) u,
$$


which is easily rearranged into the form

$$
\left[\begin{array}{c}
\dot{q} \\
\ddot{q}
\end{array}\right]=f(q, \dot{q})+g(q, \dot{q}) u,
$$

analogous to (7). (Note that $u$ denotes the input torques provided by the four actuators.) To implement the control designs of sections III-B and IV-B, we define output functions

$$
y(q):=H_{0} q-y_{d}(\theta(q)),
$$

where $\theta(q):=c q$ for some $1 \times 5$ row vector $c$ and $H_{0}$ is a $4 \times 5$ matrix satisfying

$$
\operatorname{rank}\left(\begin{array}{c}
H_{0} \\
c
\end{array}\right)=5
$$

(i.e. full rank). Our goal is to drive the controlled variables $H_{0} q$ to match $y_{d}$, which is the desired trajectory of the controlled variables as a function of $\theta(q)$, thereby imposing virtual constraints on the system. As is described in [43], we parametrize $y_{d}$ as a Bezier polynomial. Also, note that $y(q)$ has vector relative degree 2 .

The impulsive nature of walking renders this a hybrid system, with the switching surface $S$ defined by the conditions under which the swing leg impacts the ground in front of the stance leg, i.e., in (50), $h$ is the height of the foot. In particular, defining $\eta$ as in (27) results in a hybrid control system $\mathscr{H} \mathscr{C}$ that can be expressed in the form of (49) (see [49] for the specific construction). In this case, $\Delta$ describes the change in velocity due to foot strike [13].

The parameters of the Bezier polynomial, $y_{d}$, are therefore chosen so that the zero dynamics surface $Z$ (i.e. (24)) is invariant and contains an exponentially stable periodic orbit $\mathscr{O}_{Z}$ transverse to the switching surface. Therefore, by design, the assumptions of Theorem 2 are satisfied. In order to render this orbit exponentially stable for the full-order dynamics, we first obtain a symmetric positive-definite matrix $P$ by either solving the Lyapunov function (13) (with the $A$ matrix appropriately defined through choice of $K_{P}$ and $K_{D}$ ) or the Riccati equation (46), and then define $P_{\varepsilon}$ as in (23). Then employing the RES-CLF $V_{\varepsilon}(\eta)=\eta^{T} P_{\varepsilon} \eta$ and applying either the Sontag control law (38) or the pointwise min-norm control law (45) results in a closed-loop hybrid system $\mathscr{H}_{\varepsilon}$. Moreover, in both cases Theorem 2 implies that the periodic orbit $\mathscr{O}$ is exponentially stable for $\varepsilon$ sufficiently small.

The control approach described here was tested with computer simulations of four walking steps of the biped model. Plots of the associated CLF function $V_{\varepsilon}(\eta)$ for various values of $\varepsilon$ are depicted for the Sontag controller (38) in Fig. 3a and for the pointwise min-norm controller (45) in Fig. 3b. In each case, the matrix $P$ was generated using the Lyapunov equation (13). The middle graph in each figure shows slow convergence for one choice of $\varepsilon$; smaller $\varepsilon$ values result in faster convergence (top graphs), and $\varepsilon$ values which are too large result in instability (bottom graphs). In figure 4, we illustrate convergence to the periodic orbit for the coordinate $q_{1}$ for an extended version (16 steps) of the simulation depicted in the middle plot of figure $3 \mathrm{a}$. Note that saturation was not implemented on the commanded control torques for any of the simulations, and peak torques were in the range of $60 \mathrm{Nm}$ for
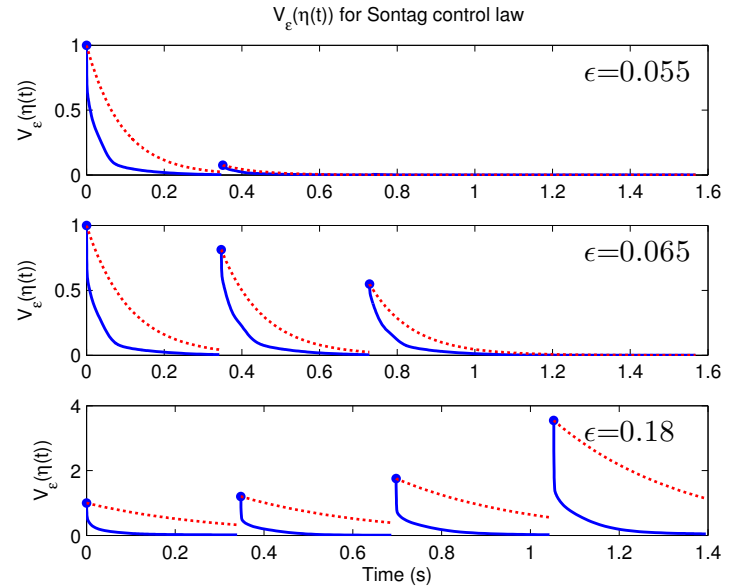

(a) $V_{\varepsilon}$ under Sontag controller.
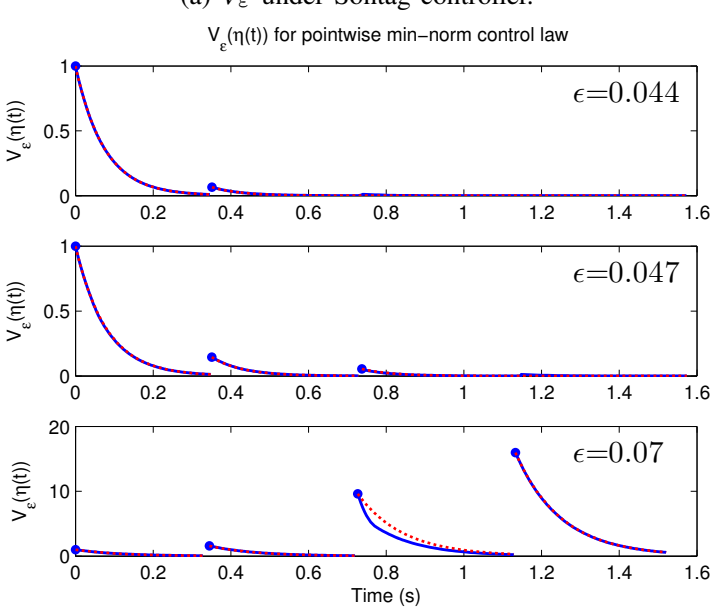

(b) $V_{\varepsilon}$ under pointwise min-norm controller.

Fig. 3: Evolution of the CLF $V_{\varepsilon}(\eta(t))=\eta^{T}(t) P_{\varepsilon} \eta(t)$ (solid line) along with the associated nominal bound (42) (dashed line) over four steps under: (Fig. 3a) the Sontag controller (38), and (Fig. 3b) the pointwise min-norm controller (45), in each case normalized so that $V_{\varepsilon}(\eta(0))=1$. Here $P_{\varepsilon}$ is based on the Lyapunov equation (13), and the values for $\varepsilon$ were chosen to demonstrate various rates of convergence (top and middle graphs) as well as instability (bottom graph). Note that for the pointwise min-norm controller, $V_{\varepsilon}$ closely follows the nominal bound as expected, only deviating in cases where zero added control (i.e. $\mu(\eta, z)=0$ ) yields performance which is better than the bound.

the min-norm controller and $100 \mathrm{Nm}$ for the Sontag controller. (See section VIII for an experimental implementation in which saturations are applied.)

\section{EXPERIMENTAL RESULTS}

Having demonstrated the effectiveness of the CLF controllers in simulation, we now describe experimental results from implementation on the robotic testbed MABEL [29], [43], a five-link bipedal walker similar to RABBIT, having a torso, two legs with revolute knees, and four actuators. (See Figure 5.) However, unlike RABBIT, MABEL has large springs in series with two of its actuators for improving 


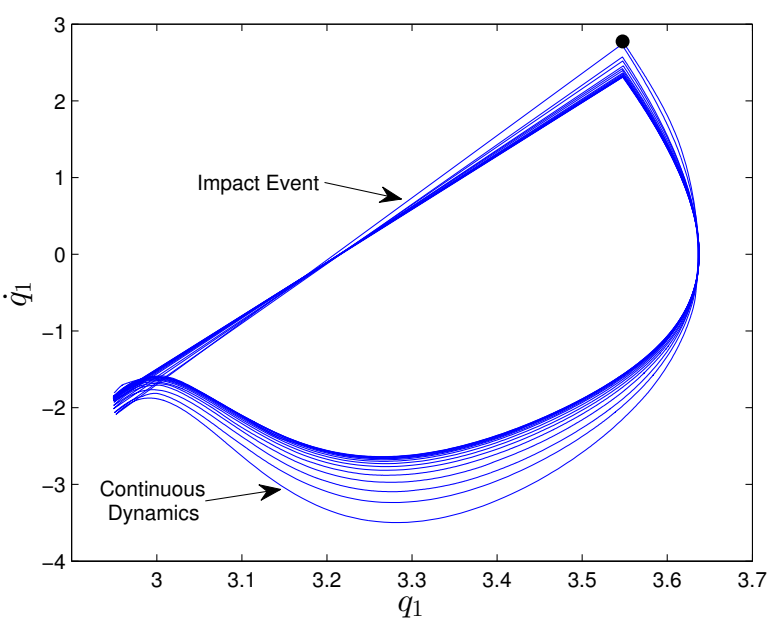

Fig. 4: Phase plot of $\left(q_{1}, \dot{q}_{1}\right)$ for an extended version (16 steps) of the simulation depicted in the middle plot of figure $3 \mathrm{a}$. The solid dot marks $\left(q_{1}, \dot{q}_{1}\right)$ at the beginning of the simulation.

energy efficiency and agility, resulting in a system model that is compliant and has a higher degree of underactuation. Although this presents several challenges for controller design, we demonstrate in this section that CLF-based controllers can be used to achieve walking gaits with MABEL while reducing undesirable oscillations of the motor torques. Note that the virtual constraints are chosen such that these controllers will not "fight" the spring, but rather preserve the natural compliant dynamics as a dominating characteristic of the closed-loop system [43].

\section{A. Control laws}

As noted, MABEL has compliance and additional degrees of underactuation. However, we can still employ the same modeling and control approach based on virtual constraints, as described in section VII. Thus our system model again takes the form (69), with $q$ coordinate labeling as described in [43] and depicted in Figure 5b, and with output functions of the form (70). For the experimental implementation, we apply the pre-control law

$$
u(q, \dot{q})=-\left(L_{g} L_{f} y(q, \dot{q})\right)^{-1} L_{f}^{2} y(q, \dot{q})+\left(L_{g} L_{f} y(q, \dot{q})\right)^{-1} \mu,
$$

which is a variation of the pre-control given by (25). Then transforming to the variables $\eta=[y, \dot{y}]^{T}$ as in (27), we have the dynamics for the transverse variables

$$
\dot{\eta}=F \eta+G \mu:=\bar{f}(\eta, z)+\bar{g}(\eta, z) \mu,
$$

where $F$ and $G$ are defined as in (29).

In what follows, we present experimental results for a CLF controller based on the pointwise min-norm controller described in section IV, and compare with experimental results based on an input-output linearizing controller with PD. The input-output linearizing controller has been employed in previous experiments with MABEL (see [43]) and takes the form (71) with $\mu$ given by

$$
\mu_{\varepsilon}(\eta, z)=\mu_{Z D}(\eta, z, \varepsilon):=-K(\varepsilon) \eta
$$

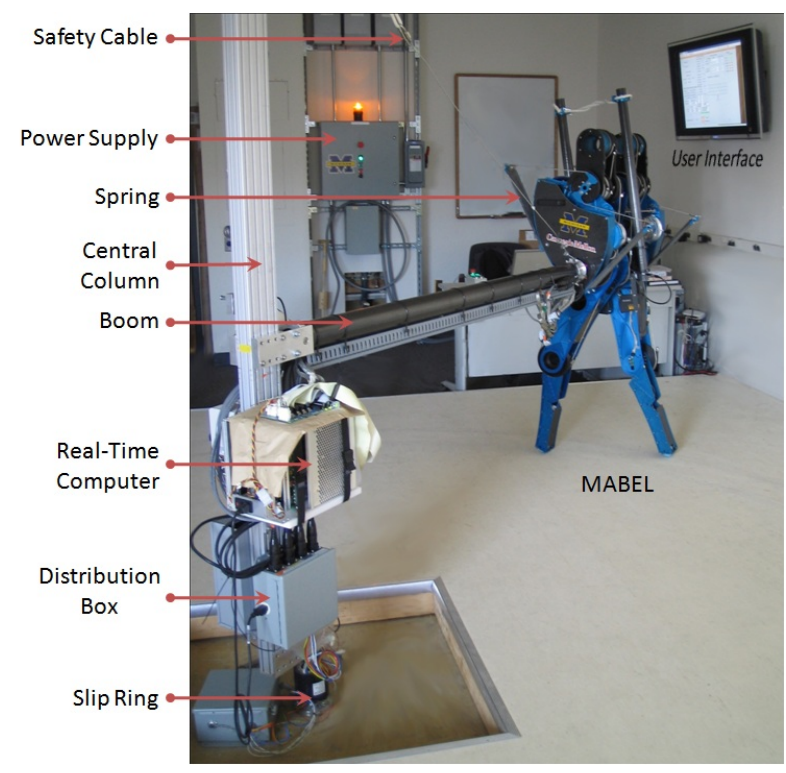

(a) MABEL experimental setup

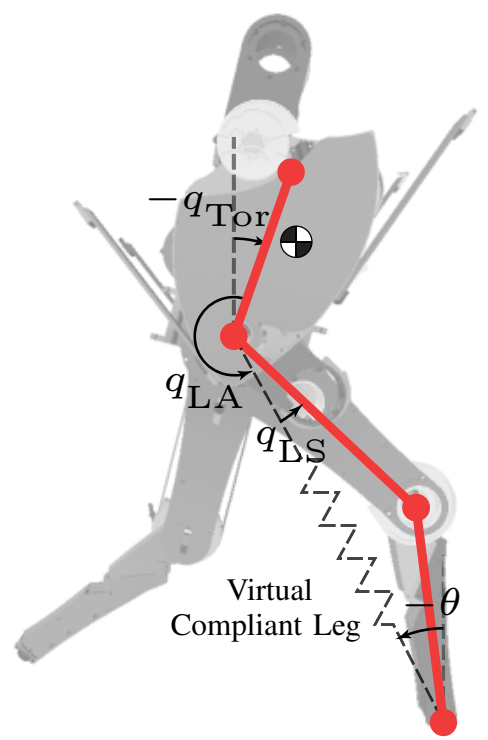

(b) Coordinates

Fig. 5: Experimental setup of the bipedal testbed MABEL and associated coordinates. (From [43].)

which is equivalent to the version given in (32). For the experiment presented here, we set $\varepsilon=0.5$ and

$$
\begin{aligned}
& K(\varepsilon)=\left[\begin{array}{ll}
\frac{1}{\varepsilon^{2}} K_{P} & \frac{1}{\varepsilon} K_{D}
\end{array}\right], \\
& K_{P}=\left[\begin{array}{cccc}
2211 & 0 & 0 & 0 \\
0 & 947 & 0 & 0 \\
0 & 0 & 2211 & 0 \\
0 & 0 & 0 & 947
\end{array}\right] \\
& K_{D}=\left[\begin{array}{cccc}
55.29 & 0 & 0 & 0 \\
0 & 8.29 & 0 & 0 \\
0 & 0 & 55.29 & 0 \\
0 & 0 & 0 & 8.29
\end{array}\right]
\end{aligned}
$$


For the CLF controller, we begin by choosing

$$
P=\left[\begin{array}{cc}
1.45556 I & .5 I \\
.5 I & .555556 I
\end{array}\right]
$$

which is the solution to the Lyapunov equation (13) where $Q$ is the $8 \times 8$ identity matrix and $A$ is given by (9) with $K_{P}=I$ and $K_{D}=1.8 I$. (Here $I$ is the $4 \times 4$ identity matrix.) Then defining our CLF $V_{\varepsilon}(\eta)$ as in (23), it follows that

$$
\begin{aligned}
& L_{\bar{f}} V_{\varepsilon}(\eta, z)=\eta^{T}\left(F^{T} P_{\varepsilon}+P_{\varepsilon} F\right) \eta, \\
& L_{\bar{g}} V_{\varepsilon}(\eta, z)=2 \eta^{T} P_{\varepsilon} G,
\end{aligned}
$$

and letting $\gamma=\frac{\lambda_{\min }(Q)}{\lambda_{\max }(P)}=0.595863$, we define

$$
\begin{aligned}
& \psi_{0, \varepsilon}(\eta, z)=L_{\bar{f}} V_{\varepsilon}(\eta, z)+\frac{\gamma}{\varepsilon} V_{\varepsilon}(\eta, z) \\
& \psi_{1, \varepsilon}(\eta, z)=L_{\bar{g}} V_{\varepsilon}(\eta, z)^{T} .
\end{aligned}
$$

Then our CLF controller is given by (71) with

$$
\mu_{\varepsilon}(\eta, z)=m_{\varepsilon}(\eta, z),
$$

where $m_{\varepsilon}\left(\eta_{\varepsilon}, z\right)$ is the pointwise min-norm control law given by (45) in terms of (76). In the experiment presented here, we set $\varepsilon=.04545$.

\section{B. Description of experiments and results}

Two experiments were conducted for the purpose of comparing the controllers just described. In Exp. 1, we employed the input-output linearizing controller (73) with gains set as described above, and obtained 85 steps of walking. For Exp. 2 , we started the robot under the same input-output linearizing controller but then transitioned to the pointwise min-norm controller (77) after 28 steps. The robot then walked for an additional 77 steps under CLF control, as can be seen in the video in [11].

In Figure 6 we display the motor torques for the stance and swing legs for 4 consecutive steps of walking under the inputoutput linearizing controller in Exp. 1, and the CLF controller in Exp. 2 respectively. (Torque saturation constraints were active in both experiments, as can be observed in Figure 6. The torque saturation for the leg shape was inadvertently raised from 10 to $12 \mathrm{Nm}$ between the experiments, but the affect on experiment comparison is negligible.) Note the reduced motor torque oscillations for the controller based on the CLF approach. Figure 7 illustrates the desired and achieved virtual constraints for the stance leg under the two controllers, displaying very close tracking of the virtual constraints by the input-output linearizing controller and rather loose tracking of the virtual constraints by the CLF-based controller. In fact, this should be expected, since the CLF-based controller always uses the minimum torque required to meet the RESCLF convergence bound (42), essentially trading off tracking performance for control efficiency as long as the required convergence bound is satisfied.

In Figure 8, we display plots of the Lyapunov function $V_{\varepsilon}$ and its calculated derivative $\dot{V}_{\varepsilon}$ for 4 consecutive steps of walking under CLF control in Exp. 2. The thin black line on the bottom plot (denoted $\dot{V}_{\varepsilon}$-online) depicts the online calculation of $\dot{V}_{\varepsilon}$ along trajectories of the partially linearized

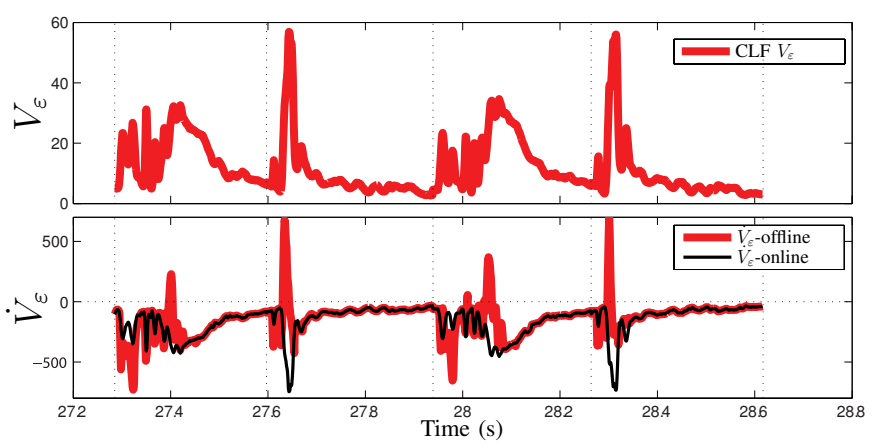

Fig. 8: Plots of the Lyapunov function and its time derivative for 4 consecutive steps of walking. (Vertical dotted lines delineate data for individual steps.) Note that the increase in $V_{\varepsilon}$ during the initial part of the gait is most likely due to torque saturation (see Figure 6b) and model uncertainty. In the plot for $\dot{V}_{\varepsilon}$, the thin black line depicts the derivative calculated in terms of the partially linearized dynamics (72) (without accounting for control saturation), which always satisfies the desired RES-CLF bound $\dot{V}_{\varepsilon} \leq-\frac{\gamma}{\varepsilon} V_{\varepsilon}$. The thick red line illustrates an offline calculation of $\dot{V}_{\varepsilon}^{\varepsilon}$ along trajectories of the full dynamics, which incorporates torque saturation.

system (72) (in terms of (75)), and does not factor in the torque saturations which are later applied to the full control $(71)^{3}$. The thick red line on the same plot (denoted $\dot{V}_{\varepsilon}$-offline) was calculated offline along trajectories of the full system dynamics (69) with the saturated versions of the control (71) with (77). The effects of these saturation constraints, along with model uncertainty, are the most likely reason that $V_{\varepsilon}$ does not exactly follow the theoretical bound (42).

\section{CONCLUSiON}

This paper presented a method for enlarging the class of controllers that exponentially stabilize periodic orbits in hybrid systems. Beginning with (hybrid) zero dynamics that contain an exponentially stable periodic, we presented a control Lyapunov function approach to designing controllers that stabilize the orbit in the full order dynamics. This was achieved by introducing a notion of control Lyapunov functions that allows for direct control of the rate of convergence to the (hybrid) zero dynamics surface: RES-CLF. Explicit motivation for this formulation was given, and an explicit means of constructing control Lyapunov functions of this form was presented. The culmination of the ideas presented in the paper was given in the main result which states that given an exponentially stable periodic orbit in a hybrid zero dynamics, the existence of a RES-CLF implies that this periodic orbit is exponentially stabilizable in the full-order dynamics. Since the theoretical results presented were originally motivated by bipedal robotic walking, they were applied in simulation to a model of the robot RABBIT. To provide further evidence of the practicality of the theoretical results, they were also applied experimentally

\footnotetext{
${ }^{3}$ The authors have also achieved experimental results (to be published separately) with a CLF-based control approach that does appropriately incorporate saturation constraints into the control calculation, making use of an online convex optimization routine.
} 


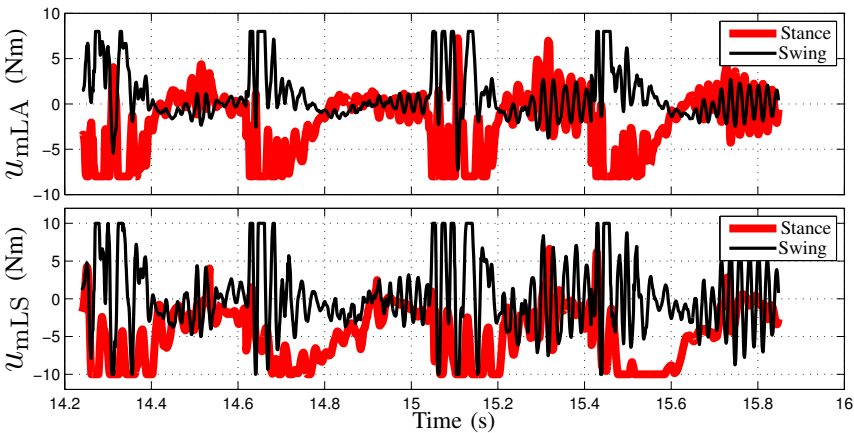

(a)

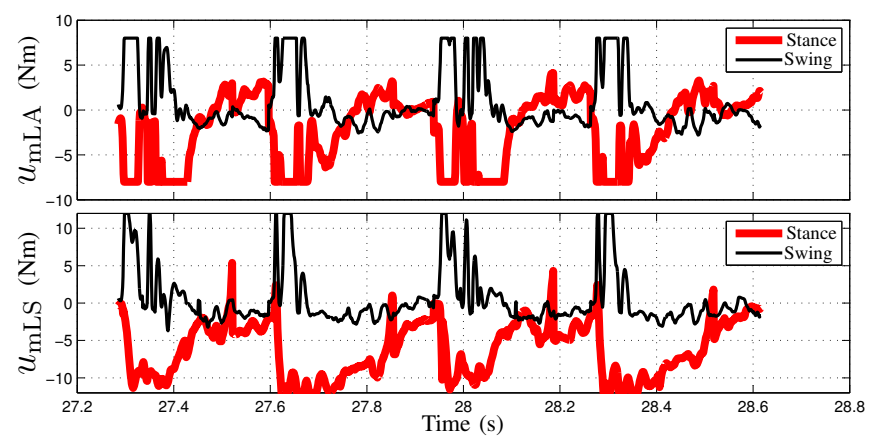

(b)

Fig. 6: Motor torques for the stance and swing legs for 4 consecutive steps of walking for (a) input-output linearizing controller in Exp. 1, and (b) CLF-based controller in Exp. 2. Note the reduced oscillations for the CLF-based controller.

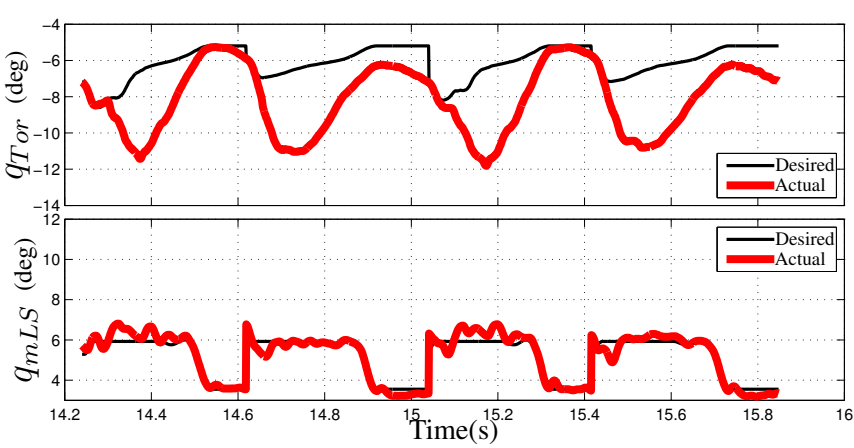

(a)

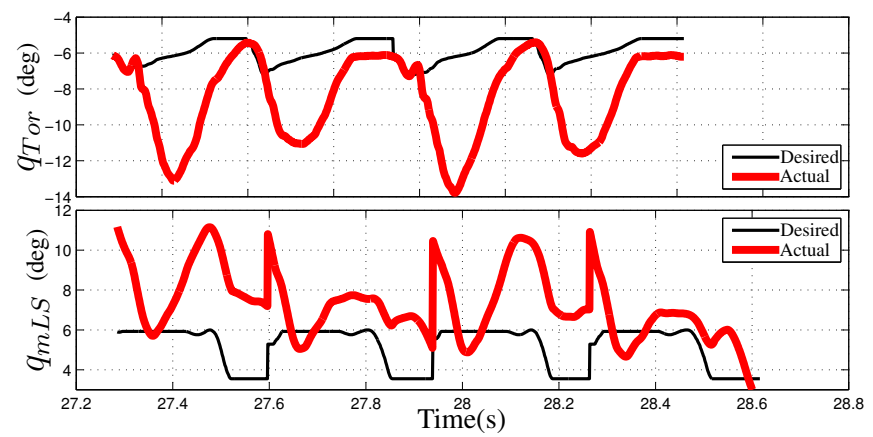

(b)

Fig. 7: Tracking for the stance-leg virtual constraints for (a) input-output linearizing controller in Exp. 1, and (b) CLF-based controller in Exp. 2. The tracking for the input-output linearizing controller is quite good, whereas the CLF-based controller always uses the minimum torque required to meet the RES-CLF convergence bound (42) and therefore does not track the virtual constraints as closely.

to MABEL. The end result is that CLFs yield better real-world controllers than previous methods. In a separate publication, it will be shown how the results of the paper allow control bounds to be explicitly incorporated into an online implementation of a RES-CLF controller.

\section{APPENDIX A}

\section{PROOF OF THEOREM 1}

Theorem 2, part (iv), of [16] is equivalent to Theorem 1, but the provided proof is incomplete. As given, the proof establishes asymptotic stability of the periodic orbit in the full-order system instead of exponential stability. The proof of exponential stability is completed here in the notation of [16]. Then, an alternative proof is given using the notation of the current paper.

\section{A. Minor addition to result in [16]}

On page 93 of [16], suppose that $V_{1}(z)$ satisfies

$$
c_{1}\|z\|_{\gamma}^{2} \leq V_{1}(z) \leq c_{1}\|z\|_{\gamma}^{2}
$$

for some $c_{1}>0$ and $c_{2}>0$. Then there exist $\alpha>0$ and $\beta>0$, such that

$$
\alpha\|z\|_{\gamma}^{2}+\alpha\|\xi\| \leq V(z, \xi) \leq \beta\|z\|_{\gamma}^{2}+\beta\|\xi\| .
$$

From the last line of the proof in [16],

$$
\dot{V}(z, \xi) \leq-k_{1}\|z\|_{\gamma}^{2}-k_{2}\|\xi\|,
$$

for $k_{1}>0$ and $k_{2}>0$. Hence,

$$
\begin{aligned}
\dot{V}(z, \xi) & \leq-\frac{k_{1}}{c_{2}} V_{1}(z)-\frac{k_{2}}{\sqrt{m_{2}}} \sqrt{V_{2}(\xi)} \\
& \leq-\mu V(z, \xi)
\end{aligned}
$$

for $\mu=\frac{k_{2}}{\sqrt{m_{2}}} \frac{1}{2 k}$ and

$$
k>\max \left\{M \frac{m_{1}}{m_{3}}, \frac{1}{2} \frac{k_{2}}{k_{1}} \frac{c_{2}}{\sqrt{m_{2}}}\right\} .
$$

Therefore,

$$
V(z(t), \xi(t)) \leq e^{-\mu t} V\left(z_{0}, \xi_{0}\right)
$$

and thus

$$
\alpha\|z(t)\|_{\gamma}^{2}+\alpha\|\xi(t)\| \leq e^{-\mu t} V\left(z_{0}, \xi_{0}\right) .
$$

It follows that

$$
\|z(t)\|_{\gamma} \leq \sqrt{\frac{1}{\alpha} e^{-\mu t} V\left(z_{0}, \xi_{0}\right)},
$$

and

$$
\|\xi(t)\| \leq \frac{1}{\alpha} e^{-\mu t} V\left(z_{0}, \xi_{0}\right) .
$$


Exponential stability is established.

\section{B. Alternative proof}

Proof: It was shown in [18] that, since $\mathscr{O}_{Z}$ is an exponentially stable periodic orbit in $Z$, there exists a Lyapunov function $V_{Z}: Z \rightarrow \mathbb{R}_{\geq 0}$ such that in a neighborhood $B_{\delta}\left(\mathscr{O}_{Z}\right)$ of $\mathscr{O}_{Z}$

$$
\begin{gathered}
k_{1}\|z\|_{\mathscr{O}_{Z}}^{2} \leq V_{Z}(z) \leq k_{2}\|z\|_{\mathscr{O}_{Z}}^{2} \\
\dot{V}_{Z}(z) \leq-k_{3}\|z\|_{\mathscr{O}_{Z}}^{2} \\
\left\|\frac{\partial V_{Z}}{\partial z}\right\| \leq k_{4}\|z\|_{\mathscr{O}_{Z}}
\end{gathered}
$$

Denote the ES-CLF $V$ by $V_{X}$ and (motivated by the construction from [23] for singularly perturbed systems) define the following Lyapunov function candidate

$$
V(x, z)=V_{Z}(z)+\frac{1}{\varepsilon} V_{X}(x)
$$

in a neighborhood $B_{\delta}(\mathscr{O})$ of $\mathscr{O}$ (where we pick $\delta>0$ to be the same $\delta$ for which $V_{Z}$ was defined). It will be shown that this function is a Lyapunov function for the orbit $\mathscr{O}$ for all $\varepsilon$ sufficiently small, i.e., that there exists an $\bar{\varepsilon}>0$ such that for all $0<\varepsilon<\bar{\varepsilon} V$ is a Lyapunov function for $\mathscr{O}$. We begin by noting that

$$
\begin{aligned}
V(x, z) & \leq k_{2}\|z\|_{\mathscr{O}_{Z}}^{2}+\frac{c_{2}}{\varepsilon}\|x\|^{2} \\
& \leq \max \left\{k_{2}, \frac{c_{2}}{\varepsilon}\right\}\left(\|z\|_{\mathscr{O}_{Z}}^{2}+\|x\|^{2}\right) \\
& \leq \max \left\{k_{2}, \frac{c_{2}}{\varepsilon}\right\}\|(x, z)\|_{\mathscr{O}}^{2}
\end{aligned}
$$

where the last equality follows from the fact that $\mathscr{O}=$ $\iota_{0}\left(\mathscr{O}_{Z}\right) \subset Z$. That is, $\|(x, z)\|_{\mathscr{O}}^{2}=\|x\|^{2}+\|z\|_{\mathscr{O}_{Z}}^{2}$. Similarly,

$$
V(x, z) \geq \min \left\{k_{1}, \frac{c_{1}}{\varepsilon}\right\}\|(x, z)\|_{\mathscr{O}}^{2} .
$$

Therefore, we need only establish that $\dot{V}(x, z) \leq$ $-\kappa_{3}\|(x, z)\|_{\mathscr{O}}^{2}$ for some $\kappa_{3}>0$. Using the fact that $q(x, z) \stackrel{=}{=}$ $q(0, z)+(q(x, z)-q(0, z))$ we have

$$
\begin{aligned}
\dot{V}(x, z) & =\left.\dot{V}_{Z}\right|_{Z}(z)+\frac{\partial V_{Z}}{\partial z}(q(x, z)-q(0, z))+\frac{1}{\varepsilon} \dot{V}_{X}(x) \\
& \leq-k_{3}\|z\|_{\mathscr{O}_{Z}}^{2}+\left\|\frac{\partial V_{Z}}{\partial z}\right\|\|q(x, z)-q(0, z)\|-\frac{c_{3}}{\varepsilon} V_{X}(x) \\
& \leq-k_{3}\|z\|_{\mathscr{O}_{Z}}^{2}+k_{4} L_{q}\|z\| \mathscr{O}_{Z}\|x\|-\frac{c_{3}}{\varepsilon} c_{1}\|x\|^{2} \\
& =-\left[\begin{array}{ll}
\|z\|_{\mathscr{O}_{Z}} & \|x\|
\end{array}\right] \Lambda\left[\begin{array}{c}
\|z\|_{\mathscr{O}_{Z}} \\
\|x\|
\end{array}\right],
\end{aligned}
$$

with $L_{q}$ the Lipschitz constant for $q$ and

$$
\Lambda=\left[\begin{array}{cc}
k_{3} & -\frac{1}{2} k_{4} L_{q} \\
-\frac{1}{2} k_{4} L_{q} & \frac{\mathrm{c}_{3}}{\varepsilon} c_{1}
\end{array}\right] .
$$

Therefore, it is necessary to pick $\varepsilon>0$ such that $\Lambda$ is symmetric positive definite. In particular, $\Lambda>0$ if $\operatorname{det}(\Lambda)>0$, that is,

$$
\frac{c_{1} c_{3} k_{3}}{\varepsilon}>\frac{1}{4} L_{q}^{2} k_{4}^{2},
$$

which is satisfied if

$$
\varepsilon<\frac{4 c_{1} c_{3} k_{3}}{L_{q}^{2} k_{4}^{2}}=: \bar{\varepsilon},
$$

wherein it follows that $\kappa_{3}=\lambda_{\min }(\Lambda)$ and we have established that $V$ is a Lyapunov function for the periodic orbit $\mathscr{O}$.

\section{APPENDIX B}

\section{RELATIONSHIP BETWEEN THEOREM 1 AND THEOREM 2}

A natural consequence of Theorem 2 is a variant of Theorem 1. That is, we consider the control system (1) as a hybrid control system with $\Delta=I$.

Corollary 1: For the system (1), let $\mathscr{O}_{Z}$ be an exponentially stable periodic orbit for the zero dynamics $\dot{z}=q(0, z)$ and assume there exists a RES-CLF $V_{\varepsilon}: X \rightarrow \mathbb{R}$. Then there exists an $\bar{\varepsilon}>0$ such that for all $0<\varepsilon<\bar{\varepsilon}$ and for all Lipschitz continuous $u_{\varepsilon}(x, z) \in K_{\varepsilon}(x, z), \mathscr{O}=\iota_{0}\left(\mathscr{O}_{Z}\right)$ is an exponentially stable hybrid periodic orbit of (41).

Remark 2: It is important to note that Corollary 1 utilizes RES-CLF rather than ES-CLF. However, the Lyapunov function (78) utilized in the proof of Theorem 1 includes a $1 / \varepsilon$ scale factor to weight the ES-CLF, and therefore both approaches rely on $\varepsilon$ to "control" the convergence to the zero dynamics surface, i.e., ensure that it is fast enough to guarantee stability of the entire system.

We finally note that the techniques used to prove Theorem 1 and Theorem 2, while similar in many respects, are actually quite different. This difference lies in the fact that for Theorem 2 a Lyapunov function on the Poincare section is used, while for Theorem 1 a Lyapunov function for the continuous dynamics (and the periodic orbit) is utilized. Extending the proof considered in the continuous case to the hybrid case is an interesting problem for future research since it would allow for a better estimate of the domain of attraction over the entire continuous dynamics rather than just the Poincaré section. Doing so would require an extension of the results in [18] to hybrid systems.

\section{REFERENCES}

[1] A. D. Ames. First steps toward underactuated human-inspired bipedal robotic walking. To appear in the IEEE International Conference on Robotics and Automation, 2012.

[2] A. D. Ames. First steps toward automatically generating bipedal robotic walking from human data. In Robotic Motion and Control, volume 422 of LNICS, pages 89-116. Springer, 2012.

[3] A. D. Ames, E. A. Cousineau, and M. J. Powell. Dynamically stable robotic walking with NAO via human-inspired hybrid zero dynamics. To appear in Hybrid Systems: Computation and Control, 2012.

[4] A. D. Ames, K. Galloway, and J. W. Grizzle. Control Lyapunov Functions and Hybrid Zero Dynamics. To appear, Proc. 51st IEEE Conf. Decision and Control, 2012.

[5] Z. Artstein. Stabilization with relaxed controls. NONLINEAR ANAL. THEORY METHODS APPLIC., 7(11):1163-1173, 1983.

[6] D.D. Bainov and P.S. Simeonov. Systems with Impulse Effects : Stability, Theory and Applications. Ellis Horwood Limited, Chichester, 1989.

[7] A. Banaszuk and J. Hauser. Feedback linearization of transverse dynamics for periodic orbits. In Decision and Control, 1994., Proceedings of the 33rd IEEE Conference on, volume 2, pages 1639 -1644 vol.2, dec 1994.

[8] S.P. Bhat and D.S. Bernstein. Continuous finite-time stabilization of the translational and rotational double integrators. IEEE Transactions on Automatic Control, 43(5):678-682, 1998.

[9] C. Chevallereau, G. Abba, Y. Aoustin, F. Plestan, E. R. Westervelt, C. Canudas-de-Wit, and J. W. Grizzle. RABBIT: A testbed for advanced control theory. IEEE Control Systems Magazine, 23(5):57-79, October 2003.

[10] R. A. Freeman and P. V. Kokotović. Robust Nonlinear Control Design. Birkhäuser, 1996.

[11] K. Galloway, K. Sreenath, A. Ames, and J. Grizzle. Walking with Control Lyapunov Functions (2012). Youtube Video. [Online]. Available: http://youtu.be/onOd7xWbGAk. 
[12] R. Goebel, R. Sanfelice, and A. Teel. Hybrid dynamical systems. IEEE Control Systems Magazine, 29(2):28-93, April 2009.

[13] J. W. Grizzle, G. Abba, and F. Plestan. Asymptotically stable walking for biped robots: Analysis via systems with impulse effects. IEEE Transactions on Automatic Control, 46(1):51-64, 2001.

[14] J. W. Grizzle, C. Chevallereau, A. D. Ames, and R. W. Sinnet. 3D bipedal robotic walking: models, feedback control, and open problems. In 8th IFAC Symposium on Nonlinear Control Systems, Bologna, Italy, 2010.

[15] J. W. Grizzle, C. H. Moog, and C. Chevallereau. Nonlinear control of mechanical systems with an unactuated cyclic variable. IEEE Transactions on Automatic Control, 30(5):559-576, May 2005.

[16] F. Grognard and C. Canudas de Wit. Design of orbitally stable zero dynamics for a class of nonlinear systems. Systems \& Control Letters, 51:89-103, 2004.

[17] W. M. Haddad, V. S. Chellaboina, and S. G. Nersesov. Impulsive and Hybrid Dynamical Systems: Stability, Dissipativity, and Control. Princeton University Press, Princeton, NJ, 2006.

[18] J. Hauser and C. C. Chung. Converse Lyapunov functions for exponentially stable periodic orbits. Systems \& Control Letters, 23:27-34, 1994.

[19] A. Isidori. Nonlinear Control Systems. Springer-Verlag, Berlin, third edition, 1995

[20] A. Isidori. Nonlinear Control Systems: An Introduction. Springer-Verlag, Berlin, Germany, 3rd edition, 1995.

[21] A. Isidori and C. Moog. On the nonlinear equivalent of the notion of transmission zeros. In Byrnes C. and A. Kurzhanski, editors, Proc. of the IIASA Conference: Modeling and Adaptive Control, pages 146-157, Berlin, 1988. Springer-Verlag.

[22] A. Jadbabaie, J. Yu, and J. Hauser. Stabilizing receding horizon control of nonlinear systems: a control lyapunov function approach. In American Control Conference, 1999. Proceedings of the 1999, volume 3, pages 1535-1539. IEEE, 1999.

[23] H.K. Khalil. Nonlinear Systems - 3rd Edition. Prentice Hall, Upper Saddle River, NJ, 2002.

[24] M. Kristic, I. Kanellakopoulos, and P. Kokotovic. Nonlinear and Adaptive Control Design. Adaptive and Learning Systems for Signal Processing, Communications and Control. Wiley, New York, 1995.

[25] B. Morris and J. W. Grizzle. Hybrid invariant manifolds in systems with impulse effects with application to periodic locomotion in bipedal robots. IEEE Transactions on Automatic Control, 54(8):1751-1764, August 2009.

[26] B. Morris and J.W. Grizzle. A restricted Poincaré map for determining exponentially stable periodic orbits in systems with impulse effects: Application to bipedal robots. In IEEE Conf. on Decision and Control, Seville, Spain, 2005.

[27] B. Morris, E. R. Westervelt, C. Chevallereau, G. Buche, and J. W. Grizzle. Fast Motions Symposium on Biomechanics and Robotics, chapter Achieving Bipedal Running with RABBIT: Six Steps toward Infinity, pages 277-297. Lecture Notes in Control and Information Sciences. Springer-Verlag, Heidelberg, Germany, 2006.

[28] A. Papachristodoulou and S. Prajna. On the construction of lyapunov functions using the sum of squares decomposition. In Decision and Control, 2002, Proceedings of the 41st IEEE Conference on, volume 3, pages 3482-3487. IEEE, 2002.

[29] Hae-Won Park, Koushil Sreenath, Jonathan W. Hurst, and Jessy W. Grizzle. Identification of a bipedal robot with a compliant drivetrain: Parameter estimation for control design. Control Systems Magazine, 31(2):63-88, April 2011.

[30] M. Reyhanoglu, A. van der Schaft, N.H. McClamroch, and I. Kolmanovsky. Dynamics and control of a class of underactuated mechanical systems. IEEE Transactions on Automatic Control, 44(9):1663-1671, 1999.

[31] R. G. Sanfelice. Control Lyapunov Functions and stabilizability of compact sets for hybrid systems. Proc. 50th IEEE Conf. Decision and Control, pages 7404-7409, 2011.

[32] S. Sastry. Nonlinear Systems: Analysis, Stability and Control. SpringerVerlag, 1999.

[33] R Sepulchre, M. Jankovic, and P. Kokotovic. Constructive Nonlinear Control. Communications and Control Engineering. Springer Verlag, London, 1997.

[34] A. Shiriaev, J.W. Perram, and C. Canudas-de Wit. Constructive tool for orbital stabilization of underactuated nonlinear systems: Virtual constraints approach. Automatic Control, IEEE Transactions on, 50(8):1164 - 1176, aug. 2005.
[35] A.S. Shiriaev, L.B. Freidovich, and S.V. Gusev. Transverse linearization for controlled mechanical systems with several passive degrees of freedom. Automatic Control, IEEE Transactions on, 55(4):893 -906, april 2010.

[36] G. Song and M. Zefran. Underactuated dynamic three-dimensional bipedal walking. In IEEE International Conference on Robotics and Automation, pages 854-859, Orlando, Florida, May 2006. IEEE Press.

[37] E. Sontag. A 'universal' contruction of Artstein's theorem on nonlinear stabilization. Systems \& Control Letters, 13:117-123, 1989.

[38] E.D. Sontag. A ŚuniversalŠconstruction of artstein's theorem on nonlinear stabilization. Systems \& control letters, 13(2):117-123, 1989.

[39] E.D. Sontag. Mathematical control theory: deterministic finite dimensional systems, volume 6. Springer, 1998.

[40] E.D. Sontag and Y. Wang. On characterizations of the input-to-state stability property. Systems \& Control Letters, 24(5):351-359, 1995.

[41] E.D. Sontag and Y. Wang. New characterizations of input-to-state stability. Automatic Control, IEEE Transactions on, 41(9):1283-1294, 1996.

[42] M.W. Spong. Energy based control of a class of underactuated mechanical systems. In Proc. of IFAC World Congress, San Francisco, CA, pages 431-435, 1996

[43] K. Sreenath, H.W. Park, I. Poulakakis, and J. W. Grizzle. A compliant hybrid zero dynamics controller for stable, efficient and fast bipedal walking on MABEL. IJRR, 30, 2011.

[44] D. Sun. A further result on an implicit function theorem for locally Lipschitz functions. Operations Research Letters, 28(4):193-198, 2001.

[45] W. Tan and A. Packard. Stability region analysis using polynomial and composite polynomial lyapunov functions and sum-of-squares programming. Automatic Control, IEEE Transactions on, 53(2):565-571, 2008.

[46] E. Wendel and A. D. Ames. Rank deficiency and superstability of hybrid systems. Nonlinear Analysis: Hybrid Systems, 6:787-805, 2012.

[47] E. Westervelt, J.W. Grizzle, and D.E. Koditschek. Hybrid zero dynamics of planar biped walkers. IEEE Transactions on Automatic Control, 48(1):42-56, January 2003.

[48] E. R. Westervelt, G. Buche, and J. W. Grizzle. Experimental validation of a framework for the design of controllers that induce stable walking in planar bipeds. International Journal of Robotics Research, 24(6):559582, June 2004.

[49] E. R. Westervelt, J. W. Grizzle, C. Chevallereau, J. H. Choi, and B. Morris. Feedback Control of Dynamic Bipedal Robot Locomotion. CRC Press, Boca Raton, 2007.

[50] H. Ye, A. N. Michel, and L. Hou. Stability theory for hybrid dynamical systems. IEEE Transactions on Automatic Control, 43(4):461-474, April 1998.

[51] W. Zhang, A. Abate, J. Hu, and M. Vitus. Exponential stabilization of dsicrete-time switched linear systems. Automatica, 45(11):2526-2536, 2009. 